\title{
ON THE STRUCTURE OF THE SOLUTION SET OF EVOLUTION INCLUSIONS WITH FRÉCHET SUBDIFFERENTIALS
}

\author{
TIZIANA CARDINALI \\ Perugia University \\ Department of Mathematics \\ Via Vanvitelli 1 \\ Perugia 06123, Italy
}

(Received May, 1998; Revised November, 1999)

In this paper we consider a Cauchy problem in which is present an evolution inclusion driven by the Fréchet subdifferential $\partial^{-} f$ of a function $f: \Omega \rightarrow R \cup\{+\infty\}$ ( $\Omega$ is an open subset of a real separable Hilbert space) having a $\varphi$-monotone subdifferential of order two and a perturbation $F: I \times \Omega \rightarrow P_{f c}(H)$ with nonempty, closed and convex values.

First we show that the Cauchy problem has a nonempty solution set which is an $R_{\delta}$-set in $C(I, H)$, in particular, compact and acyclic. Moreover, we obtain a Kneser-type theorem. In addition, we establish a continuity result about the solution-multifunction $x \rightarrow S(x)$. We also produce a continuous selector for the multifunction $x \rightarrow S(x)$. As an application of this result, we obtain the existence of solutions for a periodic problem.

Key words: Upper Semicontinuity, Hausdorff Metric, Fréchet Subdifferential, Evolution Inclusion, $\varphi$-Monotone Subdifferential of Order Two, $R_{\delta}$-Set, Continuous Selector, Periodic Problem.

AMS subject classifications: $34 \mathrm{G} 20,35 \mathrm{~K} 55$.

\section{Introduction}

The topological property that the solution set of a differential equation is an $R_{\delta}$-set in $C\left(I, R^{n}\right), I=[0, T]$, (in particular, nonempty, compact and connected) has been an object of investigation by many authors. It is known that the solution set of the Cauchy problem

$$
x^{\prime}(t)=f(t, x(t)) \text { a.e. on } I, x(0)=x_{0}
$$

where $f(\cdot, \cdot)$ is a bounded, continuous function on $I \times \mathbb{R}^{n}$, is an $R_{\delta^{-}}$set (see [2]). This result was extended recently to differential inclusions by C.J. Himmelberg-F.S. Van Vleck (cf. [13]) for autonomous systems and by F.S. DeBlasi-J. Myjak (cf. [10]) for non-autonomous systems. In a recent paper, N.S. Papageorgiou and F. Papalini 
[18] established that the solution set of an evolution inclusion, driven by a timedependent subdifferential $\partial \varphi(t, x)$ and by a convex valued perturbation term $F(t, x)$ satisfying a continuity hypothesis in the $x$-variable, is $R_{\delta}$ in $C(I, H)$, where $H$ is a separable Hilbert space.

The purpose of this paper is to study the mentioned topological property of the following Cauchy problem for evolution inclusion:

$$
\left\{\begin{array}{cc}
x^{\prime} \in-\partial^{-} f(x)+F(t, x), & \text { a.e. on } I, \\
x(0)=x_{0}, & x_{0} \in \operatorname{dom}(f),
\end{array}\right.
$$

where $\partial^{-} f$ is the Fréchet subdifferential of a function $f: \Omega \rightarrow R \cup\{+\infty\}$ ( $\Omega$ is an open subset of a real separable Hilbert space) having a $\varphi$-monotone subdifferential of order two, while $F: I \times \Omega \rightarrow P_{f c}(H)$ is a multifunction with nonempty, closed and convex values. About the problem (1), the recent existence theorems obtained in [19] and in [6] are known. In [19], Papalini also proves that the solution set for the problem (1) is path-connected. But, we want to observe that a path-connected set need not be an $R_{\delta}$ set.

In this note, we first prove two existence theorems (cf. Theorem 1 and Theorem 2) for the problem (1). Moreover, in Section 3, we obtain that the solution set $S\left(x_{0}\right)$ is a $R_{\delta}$ (cf. Theorem 3 ) if " $f$ " is a function with the properties:

(i) $\quad f$ has a $\varphi$-monotone subdifferential of order two;

(ii) $\exists r>0$ such that $c l B\left(x_{0}, r\right) \subset \Omega$ and the set $L(c)=\left\{x \in C l B\left(x_{0}, r\right)\right.$ : $\left.\|x\|^{2}+f(x) \leq c\right\}$ is compact in $H, \forall c \in R$,

(iii) $\exists \widetilde{k}, \widetilde{r}>0$ such that $f(x) \leq \widetilde{k}, \forall x \in \operatorname{dom}(f) \cap B\left(x_{0}, \tilde{r}\right)$;

(iv) $\exists N>0$ and $r^{\prime}>0$ such that $\left\|\operatorname{grad}^{-} f(x)\right\| \leq N, \forall x \in \operatorname{dom}\left(\partial^{-} f\right) \cap$ $B\left(x_{0}, r^{\prime}\right)$.

and $F: I \times \Omega \rightarrow P_{f c}(H)$ is a multifunction such that

(j) $\forall x \in \Omega, t \rightarrow F(t, x)$ is measurable;

$(j j)^{\prime \prime} \quad \forall t \in I, x \rightarrow F(t, x)$ is (u.s.c.) $)_{m}$ on $\Omega$;

(jjj) $\exists \gamma \in L^{2}\left(I, R^{+}\right):\|F(t, x)\|=\sup \{\|z\|: z \in F(t, x)\} \leq \gamma(t), \quad$ a.e. $\quad$ in $\quad I$, $\forall x \in \Omega$.

As an immediate consequence of Theorem 3, we deduce a Kneser-type theorem (cf. Corollary 1). In addition, if $F: I \times \Omega \rightarrow P_{f c}(H)$ is a multifunction satisfying conditions $(j),(j j j)$ and the following hypothesis:

$(j j)$ for a.e. $t \in I, G r F(t, \cdot)$ is sequentially closed in $\Omega \times H_{w}$ (here $H_{w}$ stands for the Hilbert space $H$ equipped with the weak topology),

we establish a continuity result about the solution-multifunction $x \rightarrow S(x)$ (cf. Theorem 4). Moreover, in order to generate a continuous selector for this solution-multifunction, we are forced to strengthen the hypothesis $(j j)$ on the orientor field " $F$ " (cf. Theorem 5) with the following condition:

$(j j)^{\prime} \quad \exists K \in L^{1}\left(I, R^{+}\right): h(F(t, x), F(t, y)) \leq k(t)\|x-y\|$, a.e. in $I, \forall x, y \in \Omega$.

Finally, we present an application of these results in the study of the existence of periodic solutions for a class of evolution inclusions involving the Fréchet subdifferential.

We want to observe that the class of proper, convex and lower semicontinuous functions is included in the class of lower semicontinuous functions with a $\varphi$-monotone subdifferential of order two (cf. [19]). 
Therefore, we can say that our theorems extend the similar results obtained in [18].

\section{Mathematical Preliminaries}

Let $X$ be a separable Banach space. We will be using the following notations:

$P_{f(c)}(X)=\{A \subseteq X: A$ nonempty, closed ( and convex) $\}$

If $(\Omega, \Sigma)$ is a measurable space, a multifunction $F: \Omega \rightarrow P_{f}(X)$ is said to be measurable, if for all $x \in X$, the function $\omega \rightarrow d(x, F(\omega))=\inf \{\|x-z\|: z \in F(\omega)\}$ is measurable. If $F(\cdot)$ is measurable, then $\operatorname{Gr} F=\{(\omega, x) \in \Omega \times X: x \in F(\omega)\} \in \Sigma \times B(X)$, with $B(X)$ being the Borel $\sigma$-field of $X$ (graph measurability), while the converse is true if we assume that there exists a complete, $\sigma$-finite measure $\mu$ defined on $\Sigma$. By $S_{F}^{p}$ $(1 \leq p \leq \infty)$ we will denote the set of all measurable selectors of $F(\cdot)$ that belong in the Lebesgue-Bochner space $L^{p}(\Omega, X)$; i.e., $S_{F}^{p}=\left\{f \in L^{p}(\Omega, X): f(\omega) \in F(\omega) \mu\right.$-a.e. $\}$. In general, this set may be empty. It is easy to check using Aumann's selection theorem (cf. [21], Theorem 5.10), that for a graph measurable multifunction $F: \Omega \rightarrow 2^{X}\{\emptyset\}$, $S_{F}^{p}$ is nonempty if and only if the function $\omega \rightarrow \inf \{\|z\|: z \in F(\omega)\}$ belongs to $L^{p}\left(\Omega, R^{+}\right)$. Recall that a subset $K$ of $L^{p}(\Omega, X)$ is decomposable if for every triple $(f, g, A) \in K \times K \times \Sigma$, we have $f \chi_{A}+g \chi_{A} \in K$, where $\chi_{A}$ denotes the characteristic function of the set $A$. Clearly, $S_{F}^{p}$ is decomposable.

A subset $A$ of $X$ is said to be an absolute retract if, given any metric space $Y$ and a closed $B \subseteq Y$ and a continuous function $f: B \rightarrow A$, there exists a continuous extension $f: Y \rightarrow A$ of $f$. Then $A$ is said to be a $R_{\delta^{-}}$set if $A=\bigcap A_{n}$ for a decreasing sequence of compact absolute retracts $A_{n}$ of $X$ (cf. [15]). $\quad n \in \mathbb{N}$

Moreover, if $\left(A_{n}\right)_{n}$ are nonempty subsets of $X$, we define

$$
s_{n \rightarrow+\infty} A_{n}=\left\{x \in X: \exists\left(x_{n}\right)_{n}, x_{n} \in A_{n}, \forall n \geq 1: x=s-\lim _{n \rightarrow+\infty} x_{n}\right\}
$$

and

$$
w_{n \rightarrow+\infty} A_{n}=\left\{x \in X: \exists\left(x_{k}\right)_{k}, x_{k} \in A_{n_{k}}, \forall k \geq 1: x=w-\lim _{k \rightarrow+\infty} x_{k}\right\}
$$

(here $w$-denotes the weak topology). We say that $A_{n}$ 's converge to $A$ in the Kuratowski-Mosco sense (denoted by $A_{n} \rightarrow A$ ) if and only if $s$-lim ${ }_{n \rightarrow+\infty} A_{n}=w \varlimsup_{n \rightarrow+\infty} A_{n}$.

Suppose $T$ is a topological space. A multifunction $F: T \rightarrow P(X)$ is said to be upper semicontinuous, (u.s.c.) $)_{t}$, if for $C \subseteq X$ nonempty closed, we have that $F^{-}(C)=\{x \in$ $X: F(x) \cap C \neq \emptyset\}$ is closed in $X$. Also $F$ is lower semicontinuous, (l.s.c. $)_{t}$, if $F^{+}(C)=\{x \in X: F(x) \subset C\}$ is closed in $X$. This definition of (l.s.c.) $t_{t}$ is equivalent to saying that if $t_{n} \rightarrow t$ in $T$ then $F(t)=\underbrace{}_{n \rightarrow+\infty} F\left(t_{n}\right)=\{x \in X$ : $\left.\lim _{n \rightarrow+\infty} d\left(x, F\left(t_{n}\right)\right)=0\right\}$.

Recall that on $P_{f}(X)$ we can define a generalized metric, known in the literature as the "Hausdorff metric", by setting for $A, B \in P_{f}(X)$,

$$
h(A, B)=\max \{\sup \{d(a, B): a \in A\}, \sup \{d(b, A): b \in B\}\}
$$

(where $d(a, B)=\inf \{\|a-b\|: b \in B\}$, similarly for $d(b, A)$ ). A multifunction $F: T \rightarrow P_{f}(X)$ is said to be Hausdorff continuous ( $H$-continuous) if it is continuous from $T$ into the metric space $\left(P_{f}(X), h\right)$. Moreover, $F$ is said to be Hausdorff upper 
semicontinuous, (u.s.c.) ${ }_{m}$, if for every $t \in T$ and for all $\varepsilon>0$, there exists $\delta>0$ such that $\left|t-t^{\prime}\right|<\delta \Rightarrow F\left(t^{\prime}\right) \subseteq F(t)+\varepsilon B_{1}$, where $B_{1}$ is the unit ball in $X$.

Let $I=[0, T]$ be furnished with the $\sigma$-finite and complete Lebesgue measure, $H$ a (real) separable Hilbert space, $\Omega$ is an open subset of $H$ and a function $f: \Omega \rightarrow R \cup\{+\infty\}$, the multifunction $\partial^{-} f: \Omega \rightarrow 2^{H}$, defined as follows

$\partial^{-} f(x)=\left\{\begin{array}{cl}\emptyset, & \text { if } f(x)=+\infty \\ \left\{\alpha \in H: \liminf _{y \rightarrow x} \frac{f(y)-f(x)-\langle\alpha, y-x\rangle}{\|y-x\|} \geq 0\right\}, & \text { if } f(x)<+\infty\end{array}\right.$

where $x$ is a fixed element of $\Omega$, is called the Fréchet subdifferential of $f$. The sets $\operatorname{dom}(f)=\{x \in \Omega: f(x)<+\infty\} \quad$ and $\operatorname{dom}\left(\partial^{-} f\right)=\left\{x \in \Omega: \partial^{-} f(x) \neq \emptyset\right\}$ are the domains of $f$ and $\partial^{-} f$ respectively. For every $x \in \operatorname{dom}\left(\partial^{-} f\right)$, we denote by $\operatorname{grad}^{-} f(x)$ the element of minimal norm of $\partial^{-} f(x)$. Recall that the values of $\partial^{-} f$ are closed and convex.

If $f: \Omega \rightarrow R \cup\{+\infty\}$ is a lower semicontinuous function, we say that $f$ has a $\varphi$ monotone subdifferential of order two if there exists a continuous map $\varphi:[\operatorname{dom}(f)]^{2} \times R^{2} \rightarrow R^{+}$such that

for every $x, y \in \operatorname{dom}\left(\partial^{-} f\right)$ and for every $\alpha \in \partial^{-} f(x)$ and $\beta \in \partial^{-} f(y)$,

we have $\langle\alpha-\beta, x-y\rangle \geq-\varphi(x, y, f(x), f(y))\left(1+\|\alpha\|^{2}+\|\beta\|^{2}\right)\|x-y\|^{2}$.

In the following, we consider the multivalued Cauchy problem:

$$
\left\{\begin{array}{cc}
x^{\prime}(t) \in-\partial^{-} f(x(t))+F(t, x(t)), & \text { a.e. on } I, \\
x(0)=x_{0}, & x_{0} \in \operatorname{dom}(f) .
\end{array}\right.
$$

By a "strong solution" of (1), we mean a function $x \in C(I, \Omega)$ such that $x(\cdot)$ is absolutely continuous on any compact subset of $(0, T)$ and with the property

(1) $\quad x(t) \in \operatorname{dom}(f)$, a.e. on $I$;

(2) $\exists h \in L^{2}(I, H)$ such that

$$
h(t) \in F(t, x(t)) \text { and } x^{\prime}(t) \in-\partial^{-} f(x(t))+h(t) \text { a.e. on } I
$$

(3) $\quad x(0)=x_{0}$.

Recall that an absolutely continuous function $x:[0, T] \rightarrow H$ is differentiable almost everywhere (see [3], Theorem 2.1) and so in problem (1) the derivative $x^{\prime}(\cdot)$ is a strong derivative.

We make the following hypothesis on the function $f$ :

$H(f)_{0}: \quad f: \Omega \rightarrow R \cup\{+\infty\}$ is a function with the properties:

(i) $f$ has a $\varphi$-monotone subdifferential of order two;

(ii) $\exists r>0$ such that $\operatorname{cl} B\left(x_{0}, r\right) \subset \Omega$ and the set $L(c)=\left\{x \in \operatorname{clB}\left(x_{0}, r\right)\right.$ :

$\left.\|x\|^{2}+f(x) \leq c\right\}$ is compact in $H, \forall c \in R$,

(iii) $\exists \widetilde{k}, \widetilde{r}>0$ such that $f(x) \leq \widetilde{k}, \forall x \in \operatorname{dom}(f) \cap B\left(x_{0}, \widetilde{r}\right)$;

(iv) $\exists N>0$ and $r^{\prime}>0$ such that $\left\|\operatorname{grad}^{-} f(x)\right\| \leq N$, $\forall x \in \operatorname{dom}\left(\partial^{-} f\right) \cap B\left(x_{0}, r^{\prime}\right)$. 
Moreover, we will need the following hypothesis on the orientor field $F$ :

$H(F)_{0}: \quad F: I \times \Omega \rightarrow P_{f c}(H)$ is a multifunction such that

(j) $\forall x \in \Omega, t \rightarrow F(t, x)$ is measurable;

$(j j)$ for a.e. $t \in I, G r F(t, \cdot)$ is sequentially closed in $\Omega \times H_{w}$ (here $H_{w}$ stands for the Hilbert space $H$ equipped with the weak topology);

$(j j j) \quad \exists \gamma \in L^{2}\left(I, R^{+}\right):\|F(t, x)\|=\sup \{\|z\|: z \in F(t, x)\} \leq \gamma(t)$, a.e. in $I, \forall x \in \Omega$.

$H(F)_{1}: \quad F: I \times \Omega \rightarrow P_{f}(H)$ is a multifunction with properties $(j),(j j j)$ and the following hypothesis

$(j j)^{\prime} \quad \exists K \in L^{1}\left(I, R^{+}\right): h(F(t, x), F(t, y)) \leq k(t)\|x-y\|$, a.e. in $I, \forall x, y \in \Omega$.

$H(F)_{2}: \quad F I \times \Omega \rightarrow P_{f c}(H)$ is a multifunction with properties $(j),(j j j)$ and the following hypothesis

$(j j)^{\prime \prime} \quad \forall t \in I, x \rightarrow F(t, x)$ is (u.s.c. $)_{m}$ on $\Omega$.

$H(F)_{3}: \quad F I \times \Omega \rightarrow P_{f}(H)$ is a multifunction with property $(j j j)$ and the following hypotheses:

$(j)^{\prime} \quad(t, x) \rightarrow F(t, x)$ has measurable graph, $(j j)^{\prime \prime \prime} \forall t \in I, x \rightarrow F(t, x)$ is (l.s.c. $)_{t}$ on $\Omega$.

First, if

$$
k=\max \left\{\|\gamma\|_{2}, \tilde{k}\right\}
$$

we observe that from our hypotheses on $f$, it is possible go find the following positive numbers:

$\exists \bar{r}>0$ such that $f(x) \geq f\left(x_{0}\right)-1, \forall x \in \operatorname{clB}\left(x_{0}, \bar{r}\right)$,

$\exists r^{*}>0$ such that $\varphi$ is bounded in the set

so, we put

$$
\left\{c l B\left(x_{0}, r^{*}\right)^{2} \cap \operatorname{dom}(f)^{2}\right\} \times\left[f\left(x_{0}\right)-1, k+k^{2} / 2\right]
$$

$$
R^{*}=\min \left\{r, \tilde{r}, r^{*}, \bar{r}, r^{\prime}\right\}
$$

(cf. $H(f),(2.3)$ and $(2.4))$. Now, we take the following version of Theorem 3.6 of Tosques (cf. [20], p. 82) into account:

Proposition 1: Let $f: \Omega \rightarrow R \cup\{+\infty\}$ be a function with a $\varphi$-monotone subdifferential of order two. Then, $\forall x_{0} \in \operatorname{dom}(f), \forall M \geq 0 \exists \bar{T}>0, \exists \widehat{r}>0$ with the property:

$$
\forall u \in \operatorname{clB}\left(x_{0}, \widehat{r}\right) \cap \operatorname{dom}(f) \text { with } f(u) \leq M
$$

$\forall T^{*}>0$ and $\forall h \in L^{2}\left(\left[0, T^{*}\right], H\right)$ with $\|h\|_{2} \leq M$, there exists a unique function $u_{h}:[0, \widehat{T}] \rightarrow \operatorname{dom}(f)$, where $\widehat{T}=\min \left\{T^{*}, \bar{T}\right\}$, that is a strong solution of the Cauchy problem

with the properties

$$
\left(P_{u}\right)_{h}\left\{\begin{array}{c}
x^{\prime} \in-\partial^{-} f(x)+h \\
x(0)=u
\end{array}\right.
$$

(i) $\quad u_{h} \in H^{1,2}([0, T], H)$ is continuous on $[0, \widehat{T}]$ and absolutely continuous on the compact subsets of $(0, \widehat{T})$;

(ii) $\quad u_{h}(t) \in \operatorname{dom}\left(\partial^{-} f\right)$ and $u_{h}^{\prime}(t) \in-\partial^{-} f\left(u_{h}(t)\right)+h(t)$ a.e. in $[0, \widehat{T}]$; 
(iii) $u_{h}(0)=u$;

(iv) $\quad u_{h} \in L^{2}([0, \widehat{T}], H)$;

(v) $\left.\quad \int_{0}^{t}\left\|u_{h}^{\prime}(s)\right\|^{2} d s \leq 2(f(u))\right)-\left(f \circ u_{h}\right)(t)+\int_{0}^{t}\|h(s)\|^{2} d s, \forall t \in[0, \widehat{T}]$;

(vi) $f \circ u_{h}$ is absolutely continuous on $[0, \widehat{T}]$;

(vii) $\left(f \circ u_{h}\right)^{\prime}(t)=\left\langle h(t)-u_{h}^{\prime}(t), u_{h}(t)\right\rangle$ a.e. in $[0, \widehat{T}]$;

(viii) $\left(f \circ u_{h}\right)\left(t_{2}\right)-\left(f \circ u_{h}\right)\left(t_{1}\right)=\int_{t_{2}}^{t_{1}}\left\langle\operatorname{grad}^{-} f\left(u_{h}(t)\right), u_{h}^{\prime}(t)\right\rangle d t, \forall t_{1}, t_{2} \in[0, \widehat{T}]$.

Remark 1: In [20], the author observed that, if $h_{1}, h_{2} \in L^{2}([0, T], H)$ and $u_{1}, u_{2}$ are strong solutions of $u_{i}^{\prime} \in-\partial^{-} f(u)+h_{i}\left(i=1,2\right.$ respectively) on $[0, T]$, then $\forall t_{0}$, $t \in[0, T]$ with $t_{0} \leq t$ we have

(ix) $\quad\left\|u_{1}(t)-u_{2}(t)\right\| \leq\left(\left\|u_{1}\left(t_{0}\right)-u_{2}\left(t_{0}\right)\right\|+\int_{t_{0}}^{t}\left\|h_{1}(s)-h_{2}(s)\right\| d s\right)$

$$
\begin{gathered}
\cdot \exp \int_{t_{0}}^{t} \varphi\left(u_{1}(s), u_{2}(s),\left(f \circ u_{1}\right)(s),\left(f \circ u_{2}\right)(s)\right)\left(1+\left\|u_{1}^{\prime}(s)-h_{1}(s)\right\|^{2}\right. \\
\left.\quad+\left\|u_{2}^{\prime}(s)-h_{2}(s)\right\|^{2}\right) d s(\text { where we put } 0 . \infty=\infty) .
\end{gathered}
$$

Fixed $x_{0} \in \operatorname{dom}(f), k$ as in $(2.2)$ and $T^{*}=T$, we can say that there exist

$$
\bar{T}=\bar{T}\left(x_{0}, k\right)>0 \text { and } \rho=\rho\left(x_{0}, k\right)>0
$$

such that $\forall u \in \operatorname{clB}\left(x_{0}, \rho\right) \cap \operatorname{dom}(f)$ with $f(u) \leq k$, and $\forall h \in L^{2}([0, T], H)$ with $\|h\|_{2} \leq k$, there exists a unique function $u_{h}:[0, \widehat{T}] \rightarrow \operatorname{dom}(f)$, where $\widehat{T}=\min \{T, \bar{T}\}$, that is a strong solution of the Cauchy problem $\left(P_{u}\right)_{h}$ with the properties $(i)$ - $($ viii) of Proposition 1.

Therefore, if we fix (cf. (2.5))

$$
R=\min \left\{\rho, \frac{R^{*}}{2}\right\}
$$

we can say that, $\forall u \in c l B\left(x_{0}, R\right) \cap \operatorname{dom}(f)$ and $\forall h \in L^{2}([0, T], H)$ with $\|h\|_{2} \leq k$, there exists a unique strong solution of the problem $\left(P_{u}\right)_{h}$, with the properties mentioned in Proposition 1 and, moreover, we have that the following conditions are satisfied:

$$
\begin{gathered}
f(x) \geq f\left(x_{0}\right)-1, \quad \forall x \in \operatorname{clB}\left(x_{0}, 2 R\right) \cap \operatorname{dom}(f), \\
L(c)=\left\{x \in \operatorname{cl} B\left(x_{0}, 2 R\right):\|x\|^{2}+f(x) \leq c\right\} \text { is compact in } H, \forall c \in R, \\
L=\sup \left\{\varphi\left(x_{1}, x_{2}, y_{1}, y_{2}\right): x_{1}, x_{2} \in \operatorname{clB}\left(x_{0}, 2 R\right) \cap \operatorname{dom}(f),\right. \\
\left.y_{1}, y_{2} \in\left[f\left(x_{0}\right)-1, k+k^{2} / 2\right]\right\} \\
f(x) \leq k, \quad \forall x \in \operatorname{clB}\left(x_{0}, R\right) \cap \operatorname{dom}(f), \\
\left\|\operatorname{grad}^{-} f(x)\right\| \leq N, \forall x \in \operatorname{clB}\left(x_{0}, 2 R\right) \cap \operatorname{dom}\left(\partial^{-} f\right),
\end{gathered}
$$

(where $\varphi:[\operatorname{dom}(f)]^{2} \times R^{2} \rightarrow R^{+}$is the mentioned continuous map that verifies $(2.1)$ ). 
Now, we choose $T^{\prime}$ such that $T^{\prime}=\frac{R^{2}}{2\left(k+1-f\left(x_{0}\right)\right)+k^{2}}$ and we define $T_{0}=$ $\min \left\{\widehat{T}, T^{\prime}\right\}$. Then we can consider the solution of the problem $\left(P_{u}\right)_{h}, \forall u \in$ $\operatorname{cl} B\left(x_{0}, R\right) \cap \operatorname{dom}(f)$, defined in $\left[0, T_{0}\right]$ (cf. $\left.H(f)\right)$.

Next let $V$ be the following set:

$$
V=\left\{h \in L^{2}\left(\left[0, T_{0}\right], H\right):\|h(t)\| \leq \gamma(t) \text { a.e. in }\left[0, T_{0}\right]\right\}
$$

and, $\forall u \in \operatorname{clB}\left(x_{0}, R\right) \cap \operatorname{dom}(f)$, we consider the function:

$$
\begin{gathered}
s_{u}: V \rightarrow C\left(\left[0, T_{0}\right], \Omega\right) \\
h \rightarrow s_{u}(h)=u_{h}
\end{gathered}
$$

where $u_{h}$ is the unique solution of the problem $\left(P_{u}\right)_{h}$.

\section{Topological Structure of the Solution Set}

In order to prove the nonemptiness and the topological structure of the solution set $S\left(x_{0}\right) \subseteq C(I, \Omega)$ of $(1)$, we will need some auxiliary results. The first is an approximation lemma which can be proved as Lemma 1 of [9] with some appropriate modifications (here the multifunction is defined in $I \times \Omega$, where $\Omega$ is an open subset of the Hilbert space $H)$.

Lemma 1: Let $F: I \times \Omega \rightarrow P_{f c}(H)$ be a multifunction satisfying the hypothesis $H(F)_{2}$, then there exists a sequence of multifunction $F_{n}: I \times \Omega \rightarrow P_{f c}(H), n \geq 1$, with the properties:

(i) $\forall n \geq 1$ and $\forall x \in \Omega$ there exist $k_{n}(x)>0$ and $\varepsilon_{n}=\varepsilon_{n}(x)>0$ such that if $x_{1}, x_{2} \in \operatorname{clB}\left(x, \varepsilon_{n}\right)=\left\{y \in \Omega:\|x-y\| \leq \varepsilon_{n}\right\}$, then $h\left(F_{n}\left(t, x_{1}\right), F_{n}\left(t, x_{2}\right)\right) \leq$ $k_{n}(x) \gamma(t)\left\|x_{1}-x_{2}\right\|$, a.e. on $I$ (i.e., a.e. on $I, F_{n}(t, \cdot)$ is locally h-Lipschitz);

(ii) $\quad F(t, x) \subseteq \ldots \subseteq F_{n}(t, x) \subseteq F_{n+1}(t, x) \subseteq \ldots, \forall(t, x) \in I \times \Omega$;

(iii) $\quad\left\|F_{n}(t, x)\right\| \leq \gamma(t)$ a.e. in $I, \forall x \in \Omega$;

(iv) $\quad F_{n}(t, x) \rightarrow F(t, x)$ as $n \rightarrow \infty$ for every $(t, x) \in I \times \Omega$

and finally there exist maps $u_{n}: I \times \Omega \rightarrow H, n \geq 1$, measurable in $I$, locally-Lipschitz in $x\left(\right.$ as $\left.F_{n}(t, \cdot)\right)$ and $u_{n}(t, x) \in F_{n}(t, x)$ for every $(t, x) \in I \times \Omega$.

Now, we prove the following result concerning the solution map $p:(u, h) \mapsto p(u, h)$, where $p(u, h)$ is the unique strong solution of the above Cauchy problem $\left(P_{u}\right)_{h}$.

Lemma 2: If hypothesis $H(f)$ holds and $R$ is the positive number defined in (2.7), then the solution map $p:\left[c l B\left(x_{0}, R\right) \cap \operatorname{dom}(f)\right] \times V \rightarrow C\left(\left[0, T_{0}\right], \Omega\right)$ is sequentially continuous by considering on $L^{2}\left(\left[0, T_{0}\right], H\right)$ the weak topology.

Proof: First, we will show that if $u \in \operatorname{clB}\left(x_{0}, R\right) \cap \operatorname{dom}(f)$, then $\forall h \in V$, we have (cf. (2.13))

$$
p(u, h)(t)=u_{h}(t) \in \operatorname{clB}(u, R), \forall t \in\left[0, T_{0}\right] .
$$

Indeed, if $t \in\left[0, T_{0}\right]$, from $(v)$ of Proposition 1 , it follows

$$
\left\|u_{h}(t)-u\right\| \leq \int_{0}^{t}\left\|u_{h}^{\prime}(s)\right\| d s \leq \sqrt{t}\left(\int_{0}^{t}\left\|u_{h}^{\prime}(s)\right\|^{2} d s\right)^{1 / 2}
$$




$$
\left.\leq \sqrt{t}\left[2(f(u))-f\left(u_{h}(t)\right)\right]+k^{2}\right)^{1 / 2},
$$

where $k$ is defined as in (2.2).

Let $\widetilde{T}=\sup \left\{t \in\left[0, T_{0}\right]:\left\|u_{h}(s)-u\right\| \leq R, \forall s \in[0, t]\right\}$, by the continuity of $u_{h}$ we have that $\tilde{T}>0$. Therefore, to obtain (3.1) it is sufficient to prove that $\widetilde{T}=T_{0}$. Indeed, if $\widetilde{T}<T_{0}$, by $(2.14),(2.8)$ and by recalling that $T_{0} \leq \frac{R^{2}}{2\left(k+1-f\left(x_{0}\right)\right)+k^{2}}$, it follows that $\left\|u_{h}(\tilde{T})-u\right\| \leq \sqrt{T_{0}}\left[2\left(k+1-f\left(x_{0}\right)\right)+k^{2}\right]^{1 / 2} \leq R$.

But this inequality, being $u_{h}$ continuous in $\widetilde{T}$, contradicts the definition of $\widetilde{T}$.

Now, by (3.1) and by (ii) of Proposition 1, we deduce that $u_{h}(t) \in \operatorname{cl} B\left(x_{0}, 2 R\right) \cap$ $\operatorname{dom}(f)$, and therefore, by using $(2.8),(3.2)$ and $(2.11)$, we obtain that

$$
f\left(u_{h}(t)\right) \in\left[f\left(x_{0}\right)-1, M_{2}\right], \forall t \in\left[0, T_{0}\right], \forall h \in V,
$$

where $M_{2}=k+\frac{k^{2}}{2}$.

Moreover, using (3.1), we claim that $s_{u}(V)$ (cf. (2.14)) is a subset of the closed and convex set

$$
K_{u}=\left\{x \in C\left(\left[0, T_{0}\right], \Omega\right): x(t) \in \operatorname{cl} B(u, R), \forall t \in\left[0, T_{0}\right]\right\} .
$$

Next, we are able to prove that $p$ is sequentially continuous by considering on $L^{2}\left(\left[0, T_{0}\right], H\right)$ the weak topology. Indeed, we consider a sequence $\left(u_{n}, h_{n}\right)_{n} \subseteq$ $\left[c l B\left(x_{0}, R\right) \cap \operatorname{dom}(f)\right] \times V$ such that $\left(u_{n}\right)_{n}$ converges to $u$ in $H$ (where $u \in$ $\left.\operatorname{clB}\left(x_{0}, R\right) \cap \operatorname{dom}(f)\right)$ and $\left(h_{n}\right)_{n}$ weakly converges to $h$ in $L^{2}\left(\left[0, T_{0}\right], H\right)$. In order to prove that $p\left(u_{n}, h_{n}\right)$ converges to $p(u, h)$ in $C\left(\left[0, T_{0}\right], \Omega\right)$, we set $x_{n}=p\left(u_{n}, h_{n}\right)$, $\forall n \in N$, and $x=p(u, h)$. Taking Remark 1 into account, we have

$$
\begin{gathered}
\left\|x_{n}(t)-x(t)\right\| \leq\left(\left\|x_{n}(0)-x(0)\right\|+\int_{0}^{t}\left\|h_{n}(s)-h(s)\right\| d s\right) \\
\left.\cdot \exp \int_{0}^{t} \varphi\left(x_{n}(s), x(s),\left(f \circ x_{n}\right)(s),(f \circ x)(s)\right)\right) \\
\cdot\left(1+\left\|x_{n}^{\prime}(s)-h_{n}(s)\right\|^{2} \mid\left\|x^{\prime}(s)-h(s)\right\|^{2}\right) d s
\end{gathered}
$$

and so, being $x_{n}(t), x(t) \in B\left(x_{0}, 2 R\right) \cap \operatorname{dom}\left(\partial^{-} f\right)$, from (vii), (viii) and (2.12), we can say that

$$
\begin{gathered}
\left\|x_{n}^{\prime}(t)\right\| \leq\left\|\operatorname{grad}^{-} f\left(x_{n}(t)\right)\right\|+\left\|h_{n}(t)\right\| \leq N+\gamma(t), \text { a.e. in }\left[0, T_{0}\right], \\
\left\|x^{\prime}(t)\right\| \leq\left\|\operatorname{grad}^{-} f(x(t))\right\|+\|h(t)\| \leq N+\gamma(t), \text { a.e. on }\left[0, T_{0}\right] .
\end{gathered}
$$

Then, we have

$$
\left\|x_{n}^{\prime}(t)-h_{n}(t)\right\|^{2} \leq N^{2}+4 \gamma^{2}(t)+4 N \gamma(t)
$$

and, therefore taking (2.2) and (2.10) into account, we can deduce that

$$
\exp \int_{0}^{t} \varphi\left(x_{n}(s), x(s),\left(f \circ x_{n}\right)(s),(f \circ x)(s)\right)\left(1+\left\|x_{n}^{\prime}(s)-h_{n}(s)\right\|^{2}\right.
$$




$$
\left.+\left\|x^{\prime}(s)-h(s)\right\|^{2}\right) d s \leq \exp \left(L\left(T_{0}+2 N^{2} T_{0}+8 k^{2}+8 N k \sqrt{T_{0}}\right)\right)=C
$$

Then, it follows that

$$
\left\|x_{n}(t)\right\| \leq\|x\|_{\infty}+2 C\left(R+k \sqrt{T_{0}}\right)=M_{1}<\infty, \forall t \in\left[0, T_{0}\right]
$$

taking the properties (3.3) and (3.7) into account, we deduce that

$$
\begin{gathered}
\left\{x_{n}(t)\right\}_{n \geq 1} \subseteq\left\{y \in H:\|y\|^{2}+f(y) \leq M_{1}^{2}+M_{2}=M_{3}\right\}=L\left(M_{3}\right) \\
\forall t \in\left[0, T_{0}\right] .
\end{gathered}
$$

Also, if $s, t \in\left[0, T_{0}\right], s \leq t, n \in N$, we have (cf. (3.6))

$$
\left\|x_{n}(t)-x_{n}(s)\right\| \leq \int_{s}^{t}\left\|x_{n}^{\prime}(\tau)\right\| d \tau \leq \sqrt{t-s}\left(N^{2} T_{0}+k^{2}+2 N k \sqrt{T_{0}}\right)=M_{4} \sqrt{t-s}
$$

from which we can say that $\left(x_{n}\right)_{n}$ is equi-Hölder continuous in $\left[0, T_{0}\right]$. Moreover, by (3.8), we have that $\left\{x_{n}(t)\right\}_{n>1}$ is included in the compact set $L\left(M_{3}\right), \forall t \in\left[0, T_{0}\right]$. Thus, by using the Arzelà-Ascoli Theorem, it follows that the set $\left\{x_{n}\right\}_{n>1}$ is relatively compact in $C\left(\left[0, T_{0}\right], \Omega\right)$. Therefore, we may assume that, by passing to a subsequence if it is necessary, $x_{n} \rightarrow y$ in $C\left(\left[0, T_{0}\right], \Omega\right)$. Finally, applying the lemma of [7], we have that $y=p(u, h)$, so we can conclude that the solution map $p$ is sequentially continuous by considering on $L^{2}\left(\left[0, T_{0}\right], H\right)$, the weak topology.

We also need the following two existence theorems for the problem (1).

Theorem 1: Let $\Omega$ be an open subset of a (real) separable Hilbert space $H$, $f: \Omega \rightarrow R \cup\{+\infty\}$ be a function satisfying $H(f)$ and $F: I \times \Omega \rightarrow P_{f c}(H)$ be a multifunction satisfying the hypothesis $H(F)_{0}$, then we can choose $T_{0}>0$ and $R>0$ as in Remark 1 such that $\forall u \in \operatorname{clB}\left(x_{0}, R\right) \cap \operatorname{dom}(f)$ the set $S(u)$ is nonempty and compact in $C\left(\left[0, T_{0}\right], \Omega\right)$.

Proof: First, we observe that the set

$$
V=\left\{h \in L^{2}\left(\left[0, T_{0}\right], H\right):\|h(t)\| \leq \gamma(t) \text { a.e. in }\left[0, T_{0}\right]\right\}
$$

is bounded, convex, closed and weakly compact in $L^{2}\left(\left[0, T_{0}\right], H\right)$. Using analogous considerations made in the first part of the proof of Lemma 2, we can say:

$$
u_{h}(t) \in \operatorname{cl} B(u, R), \quad \forall t \in\left[0, T_{0}\right], \quad \forall h \in V,
$$

by which we deduce that $u_{h}(t) \in \operatorname{clB}\left(x_{0}, 2 R\right) \cap \operatorname{dom}(f), \forall t \in\left[0, T_{0}\right], \forall h \in V$, and so we can write that $f\left(u_{h}(t)\right) \in\left[f\left(x_{0}\right)-1, k+\frac{k^{2}}{2}\right], \forall h \in V, \forall t \in\left[0, T_{0}\right]$.

Now, denoted with

$$
K_{u}=\left\{x \in C\left(\left[0, T_{0}\right], \Omega\right): x(t) \in \operatorname{clB}(u, R), \forall t \in\left[0, T_{0}\right]\right\},
$$

we have that $K_{u}$ is closed and convex and $s_{u}(V) \subseteq K_{u}$. Moreover, we claim that $s_{u}(V)$ is relatively compact. Indeed, if $u_{h} \in s_{u}(V)$, taking the property $(v)$ of Proposition 1 into account, we obtain that 


$$
\left\|u_{h}^{\prime}\right\|_{2} \leq\left[2\left(k+1-f\left(x_{0}\right)\right)+k^{2}\right]^{\frac{1}{2}}
$$

Therefore, it is possible to write the following inequality

$$
\left\|u_{h}\left(t^{*}\right)-u_{h}(t)\right\| \leq\left[2\left(k+1-f\left(x_{0}\right)\right)+k^{2}\right]^{\frac{1}{2}}\left|t^{*}-t\right|^{\frac{1}{2}}, \forall t^{*}, t \in\left[0, T_{0}\right]
$$

from which it is easy to see that $s_{u}(V)$ is uniformly equicontinuous. Moreover, for every $t \in\left[0, T_{0}\right]$, the set $W(t)=\left\{u_{h}(t) \in \Omega: u_{h} \in s_{u}(V)\right\}$ is relatively compact in $H$. Therefore, from the Arzelà-Ascoli Theorem, it follows that $s_{u}(V)$ is relatively compact in $C\left(\left[0, T_{0}\right], \Omega\right)$.

Now, we want to show that function $s_{u}$ is continuous from $V$ with the weak topo$\operatorname{logy}$ on $L^{2}\left(\left[0, T_{0}\right], H\right)$ into $K_{u}$ with the topology of $C\left(\left[0, T_{0}\right], \Omega\right)$.

First we observe that, since $s_{u}(V)$ is relatively compact in the space $C\left(\left[0, T_{0}\right], \Omega\right)$, it is sufficient to prove that $\operatorname{Gr}\left(s_{u}\right)$ is sequentially closed in $V_{w} \times s_{u}(V)$. Then let $\left(h_{n}, x_{n}\right) \in G r\left(s_{u}\right),\left(h_{n}, x_{n}\right) \rightarrow(h, x)$ in $L^{2}\left(\left[0, T_{0}\right] H\right)_{w} \times C\left(\left[0, T_{0}\right], \Omega\right)$. By the lemma of [7] we deduce that $x=s_{u}(h)$. Therefore, the function $s_{u}$ is continuous. Then, by Mazur's Theorem we can say that the set $M=c l \operatorname{conv} s_{u}(V)$ is convex and compact.

Now, let $L: V \rightarrow 2^{L^{2}\left(\left[0, T_{0}\right], H\right)}$ be the multifunction defined by

$$
L(h)=S_{F\left(\cdot, s_{u}(h)(\cdot)\right)}^{2}, \forall h \in V .
$$

It is possible to prove that the multifunction $L$ has nonempty values. To this end with fixed $h$, there exists $\left(v_{n}\right)_{n}$, where $v_{n}$ is a simple function, such that $v_{n}(t) \rightarrow s_{u}(h)(t)$ a.e. in $\left[0, T_{0}\right]$ as $n \rightarrow \infty$. Since, for a.e. $t \in\left[0, T_{0}\right], \operatorname{Gr} F(t, \cdot)$ is sequentially closed in $\Omega \times H_{w}$, we have that

$$
w_{n \rightarrow+\infty} F\left(t, v_{n}(t)\right) \subset F\left(t, s_{u}(h)(t)\right) .
$$

Since for every $n>1, v_{n}(\cdot)$ is a simple function and $F(\cdot, x)$ is measurable, we can easily check that $t \rightarrow F\left(t, v_{n}(t)\right)$ is measurable. Applying Aumann's selection theorem we can get a measurable selection $g_{n}(\cdot)$ of $F\left(\cdot, v_{n}(\cdot)\right)$ such that $\left\|g_{n}(t)\right\| \leq \gamma(t)$ a.e. on $\left[0, T_{0}\right]$, for $n>1$. By passing to a subsequence if necessary, we may assume that $g_{n} \rightarrow g$ weakly in $L^{2}\left(\left[0, T_{0}\right], H\right)$.

From Theorem 3.1 of [16] we have that $g \in S_{F\left(\cdot, s_{u}(h)(\cdot)\right)}^{2}$. Then we can say that $L$ has nonempty values. Moreover, it is easy to see that for every $h \in V$, we have $L(h)$ is a closed and convex subset of $V$. Now, if we consider in $V$ the weak topology, we claim that $L: V_{w} \rightarrow P_{f c}\left(V_{w}\right)$ is (u.s.c.) $)_{t}$. Recalling that $V_{w}$ is compact and metrizable, to show the upper semicontinuity of $L(\cdot)$, it is enough to show that $G r L$ is sequentially closed in $V_{w} \times V_{w}$. To this end, let $\left(h_{n}, f_{n}\right)_{n},\left(h_{n}, f_{n}\right) \in G r L$, with the property $\left(h_{n}, f_{n}\right) \rightarrow(h, f)$ in $V_{w} \times V_{w}$. Then, for each $n>1$, we have $s_{u}\left(h_{n}\right) \in M$ and we know that $M$ is compact in $C\left(\left[0, T_{0}\right], H\right)$. So by passing to a subsequence if necessary, we may assume that $s_{u}\left(h_{n}\right) \rightarrow q$ in $C\left(\left[0, T_{0}\right], \Omega\right)$. Now taking that $h_{n} \rightarrow h$ in $V_{w}$ into account, from the proposition of [7], we conclude that $q=s_{u}(h)$. Using again Theorem 3.1 of $[16]$, we have that $f(t) \in c l$ conv $w_{n \rightarrow+\infty}$ - $F\left(t, s_{u}\left(h_{n}\right)(t)\right) \subseteq$ $F\left(t, s_{u}(h)(t)\right)$, a.e. on $\left[0, T_{0}\right]$. Therefore, $f \in S_{F\left(\cdot, s_{u}(h)(\cdot)\right)}^{2}$. Then we can conclude that $(h, f) \in G r L$. So $L$ is (u.s.c.) $t$ as claimed.

By the fixed point theorem (see [11]), we obtain there exists $h \in V$ such that 
$h \in L(h)$. Clearly, $u_{h}$ is the desired solution of the Cauchy problem (1) (satisfying the mentioned properties of Proposition 1).

From the above proof, we see that the solution set of (1) is a subset of $M$, which we know is a compact subset of $C\left(\left[0, T_{0}\right], \Omega\right)$. So to prove the compactness of the solution set of (1) in $C\left(\left[0, T_{0}\right], \Omega\right)$, it is enough to show that it is closed in $C\left(\left[0, T_{0}\right], \Omega\right)$. So let $\left(x_{n}\right)_{n}$, where $x_{n}$ is the solution of (1) such that $x_{n} \rightarrow x$ in $C\left(\left[0, T_{0}\right], \Omega\right)$. We have:

$$
\left\{\begin{array}{c}
x_{n}^{\prime}(t) \in-\partial^{-} f\left(x_{n}(t)\right)+h_{n}(t) \text { a.e. } \\
x_{n}(0)=u
\end{array}\right.
$$

with $h_{n} \in V$. By passing to a subsequence if necessary, we may assume that $h_{n} \rightarrow h$ weakly in $V$. Again from the lemma in [7], we deduce that $x=s_{u}(h)$. Now, using Theorem 3.1 of [16], we have that $h \in S_{F(\cdot, x(\cdot))}^{2}$. Therefore, the solution set $S(u)$ is compact in $C\left(\left[0, T_{0}\right], \Omega\right)$.

Theorem 2: Let $\Omega$ be an open subset of a (real) separable Hilbert space $H$, $f: \Omega \rightarrow R \cup\{+\infty\}$ be a function satisfying $H(f)$ and $F: I \times \Omega \rightarrow P_{f}(H)$ be a multifunction satisfying the hypothesis $H(F)_{3}$, then we can choose $T_{0}>0$ and $R>0$ as in Remark 1 such that $\forall u \in \operatorname{clB}\left(x_{0}, R\right) \cap \operatorname{dom}(f), S(u)$ is a nonempty subset of $C\left(\left[0, T_{0}\right], \Omega\right)$.

Proof: Using analogous considerations made in the first part of the proof of Theorem 1, we can say that the set

$$
V=\left\{h \in L^{2}\left(\left[0, T_{0}\right], H\right):\|h(t)\| \leq \gamma(t) \text { a.e. in }\left[0, T_{0}\right]\right\}
$$

is bounded, convex, closed and weakly compact in $L^{2}\left(\left[0, T_{0}\right], H\right)$. Moreover, if $u_{h}$ is the unique solution of the problem $\left(P_{u}\right)_{h}$, we have:

$$
\begin{gathered}
u_{h}(t) \in \operatorname{clB}(u, R), \forall t \in\left[0, T_{0}\right], \quad \forall h \in V, \\
u_{h}(t) \in \operatorname{clB}\left(x_{0}, 2 R\right) \cap \operatorname{dom}(f), \quad \forall t \in\left[0, T_{0}\right], \forall h \in V, \\
f\left(u_{h}(t)\right) \in\left[f\left(x_{0}\right)-1, k+\frac{k^{2}}{2}\right], \quad \forall h \in V, \quad \forall t \in\left[0, T_{0}\right]
\end{gathered}
$$

and additionally, the set $s_{u}(V)$ is compact in $C\left(\left[0, T_{0}\right], \Omega\right)$ and the set $M=$ $c l$ conv $s_{u}(V)$ is convex and compact in $C\left(\left[0, T_{0}\right], \Omega\right)$. Now, let $R: M \rightarrow P_{f}\left(L^{1}(H)\right)$ be defined by $R(x)=S_{F(\cdot, x(\cdot))}^{1}, \forall x \in M$. From Theorem 4.1 of [16], we have that $R(\cdot)$ is (l.s.c.) $t$. Since the values of $R$ are nonempty (cf. Theorem 4.1 of [21]), closed and decomposable, we can apply Fryszkowski's selection theorem [12] and we get a continuous function $r: M \rightarrow L^{1}(H)$ such that $r(x) \in R(x)$ for every $x \in M$. Then we can observe that the function $\eta=s_{u} \circ r: M \rightarrow M$ has a fixed point, i.e. there exists $x \in M$ such that $\eta(x)=x$. Then $x$ is solution for the following problem

$$
\left\{\begin{array}{c}
x^{\prime}(t) \in-\partial^{-} f(x)+r(x) \\
x(0)=u
\end{array}\right.
$$

and so, being $r(x)(t) \in F(t, x(t))$ a.e. on $\left[0, T_{0}\right]$, we can conclude that $x \in S(u)$.

Moreover, we need to prove the following:

Lemma 3: Let $\Omega$ be an open subset of a (real) separable Hilbert space $H$, $f: \Omega \rightarrow R \cup\{+\infty\}$ be a function satisfying $H(f), x_{0} \in d o m(f)$, and $g: I \times \Omega \rightarrow H$ be a 
function such that:

( $\alpha) \quad \forall x \in \Omega, t \mapsto g(t, x)$ is measurable in $I$;

$(\alpha \alpha) \quad \exists \gamma \in L^{2}\left(I, R^{+}\right):\|g(t, x)\| \leq \gamma(t)$, a.e. on $I ; \forall x \in \Omega$,

$(\alpha \alpha \alpha)$ a.e. $t \in I, x \mapsto g(t, x)$ is locally Lipschitz (i.e., $\forall x \in \Omega$, there exists $k(x)>0$ and $\epsilon_{x}>0$ such that if $x_{1}, x_{2} \in \operatorname{clB}\left(x, \varepsilon_{x}\right)$, then $\left\|g\left(t, x_{1}\right)-g\left(t, x_{2}\right)\right\| \leq$ $k(x) \gamma(t)\left\|x_{1}-x_{2}\right\|$, a.e. on $\left.I\right)$.

Under these assumptions we can say that $\forall u \in c l B\left(x_{0}, R\right) \cap \operatorname{dom}(f)$ the following Cauchy problem:

$$
\left(P_{u}\right)_{g}\left\{\begin{array}{c}
x^{\prime} \in-\partial^{-} f(x)+g(t, x) \\
x(0)=u
\end{array}\right.
$$

has a unique strong solution defined on $\left[0, T_{0}\right]$.

Proof: Fix $u \in \operatorname{cl} B\left(x_{0}, R\right) \cap \operatorname{dom}(f)$. From $(\alpha \alpha \alpha)$, we deduce that there exists a Lebesgue null-set $M \subseteq I$ such that $\forall t \in I \backslash M$ the function $x \mapsto g(t, x)$ is locally Lipschitz.

Now, we consider the multifunction $F: I \times \Omega \rightarrow P_{f c}(H)$, where

$$
F(t, x)=\left\{\begin{array}{cc}
\{g(t, x)\}, & (t, x) \in(I-M) \times \Omega \\
\{0\}, & \text { otherwise. }
\end{array}\right.
$$

Using Theorem 2 , we have that there exists a solution of the problem $\left(P_{u}\right)_{g}$ defined on $\left[0, T_{0}\right]$. Now, we prove that there exists an interval $[0, b]$, where $\left.\left.b \in\right] 0, T_{0}\right]$, such that the solution of the problem $\left(P_{u}\right)_{g}$ is unique in this interval. In fact, if $x_{1}:\left[0, T_{0}\right] \rightarrow \Omega$ and $x_{2}:\left[0, T_{0}\right] \rightarrow \Omega$ are two strong solutions of the problem $\left(P_{u}\right)_{g}$, then we find two functions $\beta_{1}$ and $\beta_{2}$ with the properties:

$$
\begin{gathered}
\beta_{1}(t) \in \partial^{-} f\left(x_{1}(t)\right), \beta_{2}(t) \in \partial^{-} f\left(x_{2}(t)\right) \text { a.e. on }\left[0, T_{0}\right] \\
x_{1}^{\prime}(t) \in-\beta_{1}(t)+g\left(t, x_{1}(t)\right), \text { a.e. on }\left[0, T_{0}\right], \\
x_{2}^{\prime}(t) \in-\beta_{2}(t)+g\left(t, x_{2}(t)\right), \text { a.e. on }\left[0, T_{0}\right]
\end{gathered}
$$

Therefore, taking $H(f)(i)$ into account, we have

$$
\begin{gathered}
\left\langle x_{1}^{\prime}(t)-x_{2}^{\prime}(t), x_{1}(t)-x_{2}(t)\right\rangle=-\left\langle\beta_{1}(t)-\beta_{2}(t), x_{1}(t)-x_{2}(t)\right\rangle \\
+\left\langle g\left(t, x_{1}(t)\right)-g\left(t, x_{2}(t)\right), x_{1}(t)-x_{2}(t)\right\rangle \\
\leq L\left(1+\left\|\beta_{1}(t)\right\|^{2}+\left\|\beta_{2}(t)\right\|^{2}\right)\left\|x_{1}(t)-x_{2}(t)\right\|^{2} \\
+\left\langle g\left(t, x_{1}(t)\right)-g\left(t, x_{2}(t)\right), x_{1}(t)-x_{2}(t)\right\rangle .
\end{gathered}
$$

Now, using analogous considerations made previously in Lemma 2, we can say

$$
\left\|\beta_{i}(t)\right\|^{2} \leq N^{2}+4 \gamma^{2}(t)+4 N \gamma(t), \text { a.e. on }\left[0, T_{0}\right], i=1,2,
$$

and so, by $(3.10)$, let $\delta(\cdot)=L\left(1+2 N^{2}+8 \gamma^{2}(\cdot)+8 N \gamma(\cdot)\right) \in L^{1}\left(\left[0, T_{0}\right], R^{+}\right)$, we get 


$$
\begin{gathered}
\int_{0}^{t}\left\langle x_{1}^{\prime}(s)-x_{2}^{\prime}(s), x_{1}(s)-x_{2}(s)\right\rangle d s \\
\leq \int_{0}^{t} \delta(s)\left\|x_{1}(s)-x_{2}(s)\right\|^{2} d s \\
+\int_{0}^{t}\left\langle g\left(s, x_{1}(s)\right)-g\left(s, x_{2}(s)\right), x_{1}(s)-x_{2}(s)\right\rangle d s, \forall t \in\left[0, T_{0}\right] .
\end{gathered}
$$

Now, we can observe that $x_{1}(t), x_{2}(t) \in \operatorname{clB}(u, R), \forall t \in\left[0, T_{0}\right]$ and, recalling the hypothesis $(\alpha \alpha \alpha)$, we can find $k(u)>0$ and $\varepsilon_{u}>0$ such that then $\left\|g\left(t, y_{1}\right)-g\left(t, y_{2}\right)\right\| \leq$ $k(u) \gamma(t)\left\|y_{1}-y_{2}\right\|, \forall y_{1}, y_{2} \in c l B\left(u, \varepsilon_{u}\right)$, a.e. on $I$. By continuity of functions $x_{1}, x_{2}$ in the point 0 , we can say that there exists $\left.b \in] 0, T_{0}\right]$ with the property $x_{1}(t), x_{2}(t) \in$ $c l B\left(u, \varepsilon_{u}\right), \forall t \in[0, b]$. Therefore, we deduce that

$$
\left\|g\left(t, x_{1}(t)\right)-g\left(t, x_{2}(t)\right)\right\| \leq k(u) \gamma(t)\left\|x_{1}(t)-x_{2}(t)\right\|, \forall t \in[0, b] .
$$

Then, by (3.11) and (3.12) we obtain

$$
\left\|x_{1}(t)-x_{2}(t)\right\|^{2} \leq 2 \int_{0}^{t}[\delta(s)+k(u) \gamma(s)]\left\|x_{1}(s)-x_{2}(s)\right\|^{2} d s, \forall t \in[0, b],
$$

and thus, using Gronwall's inequality, we deduce that $x_{1}=x_{2}$ in $[0, b]$. In order to prove the uniqueness in the interval $\left[0, T_{0}\right]$, we set $T^{*}=\sup \left\{t \in\left[0, T_{0}\right]: x_{1}(s)=x_{2}(s)\right.$ $\forall s \in[0, t]\} . \quad$ Clearly, $T^{*}>0$ and $T^{*}=\max \left\{t \in\left[0, T_{0}\right]: x_{1}(s)=x_{2}(s) \quad \forall s \in[0, t]\right\}$. Finally, we can show that $T^{*}=T_{0}$. In fact, if by contradiction, $T^{*}<T_{0}$ we can say that the functions $x_{1}:\left[0, T_{0}\right] \rightarrow \Omega$ and $x_{2}:\left[0, T_{0}\right] \rightarrow \Omega$ are solutions of the following problem

$$
\left\{\begin{array}{c}
x \in-\partial^{-} f(x)+g(t, x) \\
x\left(T^{*}\right)=\widetilde{x} \quad\left(\text { where } \tilde{x}=x_{1}\left(T^{*}\right)=x_{2}\left(T^{*}\right)\right)
\end{array}\right.
$$

Then, thanks to the analogous argument made in order to obtain (3.13), we can show that there exists $\alpha>0$ such that

$$
\begin{aligned}
& \left\|x_{1}(t)-x_{2}(t)\right\|^{2} \\
& \quad \leq 2 \int_{T^{*}}^{t}[\delta(s)+k(x) \gamma(s)]\left\|x_{1}(s)-x_{2}(s)\right\|^{2} d s \quad \forall t \in\left[T^{*}, T^{*}+\alpha\right] .
\end{aligned}
$$

Therefore we can deduce that $x_{1}=x_{2}$ in $\left[T^{*}, T^{*}+\alpha\right]$, which is absurd by the definition of $T^{*}$. Consequently, we have that $T^{*}=T_{0}$. Hence the problem $\left(P_{u}\right)_{g}$ has a unique strong solution defined $\left[0, T_{0}\right]$.

Now, we are ready for the result on the topological structure of the solution set $S\left(x_{0}\right)$.

Theorem 3: Let $\Omega$ be an open subset of a (real) separable Hilbert space $H$, $f: \Omega \rightarrow R \cup\{+\infty\}$ be a function satisfying $H(f)$ and $F: I \times \Omega \rightarrow P_{f c}(H)$ be a multi- 
function satisfying the hypothesis $H(F)_{2}$, then we can choose $T_{0}>0$ as in Remark 1 such that $S\left(x_{0}\right)$ is nonempty and it is an $R_{\delta^{-}}$set in $C\left(\left[0, T_{0}\right], \Omega\right)$.

Proof: First, by applying Lemma 1, we can choose a sequence of multifunction $\left(F_{n}\right)_{n}, F_{n}: I \times \Omega \rightarrow P_{f c}(H), n \geq 1$ be as postulated by Lemma 1 . For fixed $n \geq 1$, we consider the following multivalued Cauchy problem

$$
\left\{\begin{array}{c}
x^{\prime}(t) \in-\partial^{-} f(x(t))+F_{n}(t, x(t)), \text { a.e. on } I \\
x(0)=x_{0} .
\end{array}\right.
$$

Moreover, by Lemma 1 , we can see that, for every $n \in N$, there exists a selection $u_{n}$ : $I \times \Omega \rightarrow H$ of the multifunction $F_{n}$ with the properties mentioned in the Lemma 1 . So, using analogous considerations made in the first part of the proof of Lemma 3, we deduce that the problem, similar to the problem (3.15), which possesses the perturbation $u_{n}$, has a solution (cf. Lemma 3 ). Hence, we can say that the set of solutions $S_{n}\left(x_{0}\right)$ of the problem (3.15) is nonempty. Now, we note that $S_{n}\left(x_{0}\right) \subseteq s_{x_{0}}(V)$, where $V$ is defined as in $(2.13)$, and $s_{x_{0}}=p\left(x_{0}, \cdot\right): V \rightarrow C\left(\left[0, T_{0}\right], \Omega\right)$. Easily (cf. Theorem 1), we can observe that the set $s_{x_{0}}(V)$ is compact in $C\left(\left[0, T_{0}\right], \Omega\right)$. Hence, in order to prove that $S_{n}\left(x_{0}\right)$ is compact, we consider $\left(x_{m}\right)_{m}, x_{m} \in S_{n}\left(x_{0}\right), m \geq 1$, and $x_{m} \rightarrow x$ in $C\left(\left[0, T_{0}\right], \Omega\right)$ as $m \rightarrow \infty$. We have that $x_{m}=s_{x_{0}}\left(f_{m}\right)$ with $f_{m} \in$ $S_{F_{n}\left(\cdot, x_{m}(\cdot)\right)^{2}}^{2}$ We may assume that $f_{m} \rightarrow f^{*}$ weakly in $L^{2}\left(\left[0, T_{0}^{0}\right], H\right)$ and $f^{*} \in V$, being $V$ weakly closed in $L^{2}\left(\left[0, T_{0}\right], H\right)$. Moreover, $f^{*} \in S_{F_{n}(\cdot, x(\cdot))^{*}}^{2}$ In fact, Lemma 2 provides that $x_{m} \rightarrow s_{x_{0}}\left(f^{*}\right)=x$ in $C\left(\left[0, T_{0}\right], \Omega\right)$, so applying the convergence theorem (cf. [1]), we have that $f^{*}(t) \in F_{n}(t, x(t))$, a.e. in $\left[0, T_{0}\right]$. Thus $S_{n}\left(x_{0}\right)$ is closed, hence compact in $C\left(\left[0, T_{0}\right], \Omega\right)$.

We also claim that, for every $n \geq 1, S_{n}\left(x_{0}\right)$ is contractible. To check this property, we consider, given $r \in\left[0, T_{0}\right)$ and $x \in S_{n}\left(x_{0}\right)$, the following problem

$$
\left\{\begin{array}{c}
z^{\prime} \in-\partial^{-} f(z)+u_{n}(t, z), \text { a.e. on }[r, T] \\
z(r)=x(r)
\end{array}\right.
$$

where $u_{n}$ is the mentioned selector of $F_{n}$. The problem (3.16) has a unique solution. In fact, by denoting with $g_{n}: I \times \Omega \rightarrow H$ the function defined $g_{n}(\tau, z)=u_{n}(\tau+r, z)$ $\left(=u_{n}(t, z)\right), \forall \tau \in[0, T-r], \forall z \in \Omega$, the problem (3.16) can be rewritten in this way:

$$
\left\{\begin{array}{c}
z^{\prime} \in-\partial^{-} f(z)+g_{n}(\tau, z), \text { a.e. on }[0, T-r] \\
z(0)=x(0)=x_{0} .
\end{array}\right.
$$

By Lemma 3 , we can $\underset{\sim}{\sim}$ educe that this problem has a unique solution defined in the interval $\left[0, \widetilde{T}_{0}\right]$, where $\widetilde{T}_{0}=\min \left\{T-r, \bar{T}, T^{\prime}\right\}$. Therefore, since $T_{0}-r<\widetilde{T}_{0}$, the problem $(3.16)^{\prime}$ has a unique solution $\bar{x}$ defined in $\left[0, T_{0}-r\right]$. Now setting $\tilde{x}(t)=$ $\bar{x}(t-r)=\bar{x}(\tau), t \in\left[r, T_{0}\right]\left(\tau \in\left[0, T_{0}-r\right]\right)$, we have that $\widetilde{x}:\left[r, T_{0}\right] \rightarrow \Omega$ is the unique solution of the problem (3.16) such that

$$
u_{n}(t, \tilde{x}(t)) \in F_{n}(t, \tilde{x}(t)), \text { a.e. in }\left[r, T_{0}\right]
$$


$\widetilde{x}$ is continuous on $\left[r, T_{0}\right]$ and absolutely continuous on the compact subsets of $] r, T_{0}[$;

$$
\tilde{x}(t) \in \operatorname{clB}\left(x_{0}, 2 R\right), \quad \forall t \in\left[r, T_{0}\right]
$$

(where $R$ is chosen as in Remark 1)

$$
\begin{gathered}
f(\tilde{x}(t)) \in\left[f\left(x_{0}\right)-1, k+\frac{k^{2}}{2}\right], \quad \forall t \in\left[r, T_{0}\right] ; \\
\left\|\tilde{x}^{\prime}(t)\right\|^{2} \leq\left\|\operatorname{grad}^{-} f(\bar{x}(t-r))\right\|+\left\|u_{n}(t-r, \bar{x}(t-r))\right\| \\
\leq N+\gamma(t), \quad \forall t \in\left[r, T_{0}\right] .
\end{gathered}
$$

Now, we denote with $z(r, x)(\cdot) \in C\left(\left[r, T_{0}\right], \Omega\right)$ the unique solution of the problem (3.16). For $r=T_{0}$, we set $z\left(T_{0}, x\right)=x$. So, we can define the function $h:\left[0, T_{0}\right] \times S_{n}\left(x_{0}\right) \rightarrow S_{n}\left(x_{0}\right)$ by

$$
h(r, x)(t)=\left\{\begin{array}{cc}
x(t) & \text { for } 0 \leq t \leq r \\
z(r, x)(t) & \text { for } r \leq t \leq T_{0}
\end{array}\right.
$$

Evidently, $h(r, x)(0)=x(0)=x_{0}$. On the other hand, $h(0, x)=z(0, x)=z_{0}$, with $z_{0} \in C\left(\left[0, T_{0}\right], \Omega\right)$ being the unique solution of

$$
\left\{\begin{array}{c}
z^{\prime} \in-\partial^{-} f(z)+u_{n}(t, z) \text { a.e. on }\left[0, T_{0}\right] \\
z(0)=x(0)=x_{0}
\end{array}\right.
$$

Moreover, $h\left(T_{0}, x\right)=z\left(T_{0}, x\right)=x$.

If we can show that $h(\cdot, \cdot)$ is continuous, we will have established the contractibility of $S_{n}\left(x_{0}\right)$ in $C\left(\left[0, T_{0}\right], \Omega\right)$. To this end, let $\left\{\left(r_{m}, x_{m}\right)\right\}_{m} \subseteq\left[0, T_{0}\right] \times S_{n}\left(x_{0}\right)$, with $\left(r_{m}, x_{m}\right) \rightarrow(r, x)$ in $\left[0, T_{0}\right] \times S_{n}\left(x_{0}\right)$. We consider two distinct cases:

Case 1: $r_{m}>r$, for every $m \geq 1$ :

Let

$$
h\left(r_{m}, x_{m}\right)(t)=\left\{\begin{array}{cr}
x_{m}(t) & \text { for } 0 \leq t \leq r_{m} \\
z\left(r_{m}, x_{m}\right)(t) & \text { for } r_{m} \leq t \leq T_{0}
\end{array}\right.
$$

we put $v_{m}=h\left(r_{m}, x_{m}\right)$. Evidently, $v_{m} \in S_{n}\left(x_{0}\right), m \geq 1$, and so by passing to a subsequence if necessary, ew may assume that $v_{m} \rightarrow v$ in $C\left(\left[0, T_{0}\right], \Omega\right)$. From the definition of $h(\cdot, \cdot)$, we see that, for $t \in[0, r]$, we have $v(t)=x(t)$. Let $y \in C\left(\left[0, T_{0}\right], \Omega\right)$ be the unique solution of

$$
\left\{\begin{array}{c}
y^{\prime}(t) \in-\partial^{-} f(y(t))+u_{n}(t, v(t)), \text { a.e. on }\left[r, T_{0}\right] \\
y(r)=x(r)(=v(r)) .
\end{array}\right.
$$

Let $N \geq 1$, we can say that there exists $m_{N} \in N$ such that for all $m \geq m_{N}$ we have $r_{m}<r_{N}$. Then, for all $m \geq m_{N}$ we obtain that $v_{m}^{\prime}(t) \in-\partial^{-} f\left(v_{m}(t)\right)+$ 
$u_{n}\left(t, v_{m}(t)\right)$ a.e. on $\left[r_{N}, T_{0}\right]$ (where $\left.\left[r_{N}, T_{0}\right] \subseteq\left[r_{m}, T_{0}\right]\right)$. Then, we find two functions $\alpha_{m}$ and $\alpha$ with the properties:

$$
\begin{gathered}
\alpha_{m}(t) \in \partial^{-} f\left(v_{m}(t)\right), \alpha(t) \in \partial^{-} f(y(t)), \text { a.e. on }\left[r_{N}, T_{0}\right] \\
\alpha_{m}(t)=-v_{m}^{\prime}(t)+u\left(t, v_{m}(t)\right), \alpha(t)=-y^{\prime}(t)+u_{n}(t, v(t)), \text { a.e. on }\left[r_{N}, T_{0}\right] .
\end{gathered}
$$

By $(i x)$ of Remark 1, we deduce

$$
\begin{aligned}
& \left\|y(t)-v_{m}(t)\right\| \\
& \quad \leq\left(\left\|y\left(r_{N}\right)-v_{m}\left(r_{N}\right)\right\|+\int_{r_{N}}^{t}\left\|u_{n}(s, v(s))-u_{n}\left(s, v_{m}(s)\right)\right\| d s\right) \\
& \quad \exp \int_{r_{N}}^{t} \varphi\left(y(s), v_{m}(s),(f \circ y)(s),\left(f \circ v_{m}(s)\right)\right)\left(1+\|\alpha(s)\|^{2}+\left\|\alpha_{m}(s)\right\|^{2}\right) d s .
\end{aligned}
$$

Now, taking $v_{m}(t), y(t) \in \operatorname{clB}\left(x_{0}, 2 R\right)$, and $f\left(v_{m}(t)\right), \quad f(y(t)) \in\left[f\left(x_{0}\right)-1, k+\frac{k^{2}}{2}\right]$, $\forall t \in\left[r_{N}, T_{0}\right]$ into account, using analogous considerations made previously in Lemma 2 , we have

$$
\begin{gathered}
\exp \int_{r_{N}}^{t} \varphi\left(y(s), v_{m}(s),(f \circ y)(s),\left(f \circ v_{m}(s)\right)\right)\left(1+\|\alpha(s)\|^{2}+\left\|\alpha_{m}(s)\right\|^{2}\right) d s \\
\leq \exp \left(L T_{0}\left(1+2 N^{2}\right)+8 k^{2} L+8 N L k \sqrt{T_{0}}\right)=C
\end{gathered}
$$

then, we can say that

$$
\begin{aligned}
& \left\|y(t)-v_{m}(t)\right\| \\
& \leq C\left[\left\|y\left(r_{N}\right)-v_{m}\left(r_{N}\right)\right\|+\int_{r_{N}}^{t}\left\|u(s, v(s))-u_{n}\left(s, v_{m}(s)\right)\right\| d s\right], \forall t \in\left[r_{N}, T_{0}\right] .
\end{aligned}
$$

Applying the limit $m \rightarrow \infty$, we get that

$$
\|y(t)-v(t)\| \leq C\left\|y\left(r_{N}\right)-v\left(r_{N}\right)\right\| \text {, for } t \in\left[r_{N}, T_{0}\right]
$$

Note that as $N \rightarrow \infty$ we have $y\left(r_{N}\right) \rightarrow x(r)$ and $v\left(r_{N}\right) \rightarrow v(r)=x(r)$. Since $N \geq 1$ was arbitrary, we conclude that $y(t)=v(t)$ for $t \in\left[r, T_{0}\right]$. Hence $v=h(r, x)$ and so $h\left(r_{m}, x_{m}\right) \rightarrow h(r, x)$ in $C\left(\left[0, T_{0}\right], \Omega\right)$ as $m \rightarrow \infty$.

Case 2: $\quad r_{m} \leq r$, for every $m \geq 1$. Now, for all $t \in[0, r[$, there exists a natural number $\bar{m}$ such that for all $m \geq \bar{m}$ we have $r_{m}>t$. Keeping the notation introduced in the analysis of Case 1 , we see that $v(t)=x(t)$ for $t \in[0, r]$.

Moreover, the same arguments as in Case 1 give us that

$$
\left\|y(t)-v_{m}(t)\right\| \leq C\left[\left\|y(r)-v_{m}(r)\right\|+\int_{r}^{t}\left\|u_{n}(s, v(s))-u_{n}\left(s, v_{m}(s)\right)\right\| d s\right]
$$

and by applying the limit $m \rightarrow \infty$, we obtain 


$$
\|y(t)-v(t)\| \leq C\|y(r)-v(r)\| \text { for } t \in\left[r, T_{0}\right] .
$$

But $y(r)=x(r)=v(r)$. So $y(t)=v(t)$ for $t \in\left[r, T_{0}\right]$. Hence, $v=h(r, x)$ and so again we have $h\left(r_{m}, x_{m}\right) \rightarrow h(r, x)$ in $C\left(\left[0, T_{0}\right], \Omega\right)$ as $m \rightarrow \infty$.

In general, we can always find a subsequence $\left\{r_{m}\right\}_{m}>1$ satisfying Case 1 or Case 2. Thus we have proved the continuity of the map $h(\cdot, \cdot)$. So, for every $n \geq 1$, $S_{n}\left(x_{0}\right)$ is compact and contractible in $C\left(\left[0, T_{0}\right], \Omega\right)$. To finish the proof we show that $S\left(x_{0}\right)=\bigcap_{n \in N} S_{n}\left(x_{0}\right)$. Clearly $S\left(x_{0}\right) \subseteq \bigcap_{n \in N} S_{n}\left(x_{0}\right)$. Let $x \in \bigcap_{n \in N} S_{n}\left(x_{0}\right)$. Then by definition $x \stackrel{n \in N}{=} s_{x_{0}}\left(h_{n}\right)$ with $h_{n} \in S_{F}^{2}(\cdot, x(\cdot)), n \geq 1$. Evidently $\left\{h_{n}\right\}_{n \geq 1}$ is bounded in $L^{2}\left(\left[0, T_{0}\right], H\right)$. So, by passing to a subsequence if necessary, we may assume that $h_{n} \rightarrow h$ weakly in $L^{2}\left(\left[0, T_{0}\right], H\right)$. From Theorem 3.1 of [16], we have that

$$
h(t) \in \overline{\mathrm{conv}} \underset{n \rightarrow \varlimsup_{n \rightarrow+\infty}}{ } F_{n}(t, x(t)) \subseteq F(t, x(t)), \text { a.e. on }\left[0, T_{0}\right],
$$

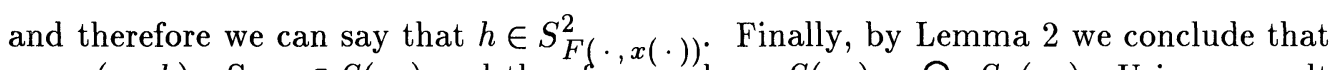
$x=p\left(x_{0}, h\right)$. So $x \in S\left(x_{0}\right)$ and therefore we have $S\left(x_{0}\right)=\bigcap_{n \in N} S_{n}\left(x_{0}\right)$. Using a result of [15], we conclude that $S\left(x_{0}\right)$ is a $R_{\delta^{-}}$set in $C\left(\left[0, T_{0}\right], \Omega\right)$.

Being the evolution map " $e$ " defined by $e(x, t)=x(t), \forall(x, t) \in C\left(\left[0, T_{0}\right], \Omega\right) \times$ $\left[0, T_{0}\right]$, a continuous function, we have as an immediate consequence of Theorem 3 above, the following Kneser-type theorem for (1).

Corollary 1: If hypotheses $H(f)$ and $H(F)_{2}$ hold, then for every $t \in\left[0, T_{0}\right]$, the set $R(t)=S\left(x_{0}\right)(t)=\left\{x(t): x \in S\left(x_{0}\right)\right\}$ (the reachable set at time $t \in I$ ) is compact and connected in $H$.

In what follows we obtain, as a consequence of Theorem 1 and Lemma 2, a continuity result about the solution-multifunction $x \mapsto S(x)$.

Theorem 4: If hypotheses $H(f)$ and $H(F)_{0}$ hold, then there exist $T_{0}>0$ and $R>0$, chosen as in Remark 1 , such that the multifunction $S: \operatorname{dom}(f) \cap \operatorname{clB}\left(x_{0}, R\right) \rightarrow$ $P_{k}\left(C\left(\left[0, T_{0}\right], \Omega\right)\right)$ is (u.s.c. $)_{t}$.

Proof: The set $S(x)$ is nonempty and compact in $C\left(\left[0, T_{0}\right], \Omega\right)$ for every $x \in \operatorname{dom}(f) \cap \operatorname{clB}\left(x_{0}, R\right)$ (see, Theorem 1). Now we need to show that given $C \subseteq$ $\left.C\left(\left[0, T_{0}\right], \Omega\right)\right)$ nonempty and closed the set, $S^{-}(C)=\left\{x \in \operatorname{dom}(f) \cap \operatorname{clB}\left(x_{0}, R\right)\right.$ : $S(x) \cap C \neq \emptyset\}$ is closed in $\operatorname{dom}(f) \cap \operatorname{clB}\left(x_{0}, R\right) \subseteq \Omega$. To this end, let $u_{n} \in S^{-}(C)$, $n \geq 1$. For each $n \geq 1$, let $x_{n}=p\left(u_{n}, h_{n}\right), h_{n} \in S_{F\left(\cdot, x_{n}(\cdot)\right)}^{2}$. Since $\left\{h_{n}\right\}_{n}$ is bounded in $L^{2}\left(\left[0, T_{0}\right], H\right)$ (cf. hypothesis $\left.(j j j)\right)$, by passing to a subsequence if necessary, we may assume that $h_{n} \rightarrow h$ weakly in $V \subseteq L^{2}\left(\left[0, T_{0}\right], H\right)$. From Lemma 2 we have that $p\left(u_{n}, h_{n}\right) \rightarrow p(u, h)$ in $\left.C\left(\left[0, T_{0}\right], \Omega\right)\right)$. Now let $x=p(u, h)$, from hypothesis $H(F)_{0}(j j)$

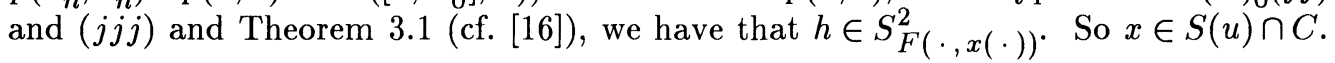

Since $u \in \operatorname{dom}(f) \cap \operatorname{cl} B\left(x_{0}, R\right)$, we can conclude that $u \in S^{-}(C)$. Therefore $S(\cdot)$ is (u.s.c.) .

Next we will generate a continuous selector for the multifunction $x \mapsto S(x)$. For this we will need the hypothesis $H(F)_{1}$ on the orientor field $F$.

Theorem 5: If hypotheses $H(f)$ and $H(F)_{1}$ hold and the set dom $(f)$ is a closed subset of $\Omega$, then, for all $\eta>0$ such that $\operatorname{cl} B\left(x_{0}, \eta\right) \subseteq B\left(x_{0}, R\right)$ there exists

$u: \operatorname{dom}(f) \cap \operatorname{clB}\left(x_{0}, \eta\right) \rightarrow C\left(\left[0, T_{0}\right], \Omega\right)$ a continuous map such that $u(\xi) \in S(\xi)$ for every $\xi \in \operatorname{dom}(f) \cap \operatorname{cl} B\left(x_{0}, \eta\right)$.

Proof: Let $x_{0}(\xi)(\cdot) \in C\left(\left[0, T_{0}\right], \Omega\right)$ be the unique solution of the evolution equation (cf. Proposition 1) 


$$
\left\{\begin{array}{c}
x^{\prime} \in-\partial^{-} f(x) \\
x(0)=\xi \in \operatorname{dom}(f) \cap \operatorname{clB}\left(x_{0}, \eta\right)
\end{array}\right.
$$

Let $R_{0}: \operatorname{dom}(f) \cap \operatorname{clB}\left(x_{0}, \eta\right) \rightarrow P_{f}\left(L^{1}\left(\left[0, T_{0}\right], H\right)\right)$ be defined $R_{0}(\xi)=S_{F\left(\cdot, x_{0}(\xi)(\cdot)\right)}^{1}$, for every $\xi \in \operatorname{dom}(f) \cap \operatorname{cl} B\left(x_{0}, \eta\right)$. Now we can observe, using analogous considerations made in the first part of Lemma 2 , that $R_{0}(\cdot)$ is $h$-Lipschitz and, moreover, that its values are nonempty, closed and decomposable. So we can apply Theorem 3 of [4] and we obtain $r_{0}: \operatorname{dom}(f) \cap \operatorname{clB}\left(x_{0}, \eta\right) \rightarrow L^{1}\left(\left[0, T_{0}\right], H\right)$ a continuous map such that $r_{0}(\xi) \in R_{0}(\xi)$, for every $\xi \in \operatorname{dom}(f) \cap \operatorname{clB}\left(x_{0}, \eta\right)$.

Let $x_{1}(\xi)(\cdot) \in C\left(\left[0, T_{0}\right], \Omega\right)$ be the unique solution of

$$
\left\{\begin{array}{c}
x^{\prime} \in-\partial^{-} f(x)+r_{0}(\xi) \\
x(0)=\xi \in \operatorname{dom}(f) \cap \operatorname{clB}\left(x_{0}, \eta\right) .
\end{array}\right.
$$

We claim that, for every fixed $\xi \in \operatorname{dom}(f) \cap \operatorname{cl} B\left(x_{0}, \eta\right)$, by induction we can generate two sequences $\left\{x_{n}(\xi)(\cdot)\right\}_{n \geq 0} \subseteq C\left(\left[0, T_{0}\right], \Omega\right)$ and $\left\{r_{n}(\xi)(\cdot)\right\}_{n \geq 0} \subseteq$ $L^{2}\left(\left[0, T_{0}\right], H\right)$ satisfying the following properties:

(a) $\quad x_{n}(\xi)(\cdot) \in C\left(\left[0, T_{0}\right], \Omega\right)$ is the unique solution of

$$
\left\{\begin{array}{c}
x^{\prime}(t) \in-\partial^{-} f(x(t))+r_{n-1}(\xi)(t) \text { a.e. in }\left[0, T_{0}\right] \\
x(0)=\xi, \forall n \geq 1
\end{array}\right.
$$

(b) $\quad \xi \rightarrow r_{n}(\xi)$ is continuous from $\operatorname{dom}(f) \cap \operatorname{clB}\left(x_{0}, \eta\right)$ into $L^{1}\left(\left[0, T_{0}\right], H\right), \forall n \geq 0$;

(c) $\quad r_{n}(\xi)(t) \in F\left(t, x_{n}(\xi)(t)\right)$ a.e. on $\left[0, T_{0}\right]$, for every $\xi \in \operatorname{dom}(f) \cap c l B\left(x_{0}, \eta\right)$, $\forall n \geq 0$;

(d) $\quad\left\|r_{n}(\xi)(t)-r_{n-1}(\xi)(t)\right\| \leq k(t) \beta_{n}(t)$ a.e. on $\left[0, T_{0}\right], \forall n \geq 1$, with

$$
\beta_{n}(t)=\int_{0}^{t} C^{n} \gamma(s) \frac{(\theta(t)-\theta(s))^{n-1}}{(n-1) !} d s+T_{0}\left(\sum_{k=0}^{n} \frac{\varepsilon}{2^{k+1}}\right) \frac{(\theta(t))^{n-1}}{(n-1) !}
$$

with $\varepsilon>0$ and $\theta(t)=\int_{0}^{t} k(s) d s$.

Our first purpose is to prove that we are able to find the functions $x_{1}(\xi)(\cdot)$ and $r_{0}(\xi)(\cdot)$ satisfying the properties $(a)-(d)$. Evidently the fixed function $x_{1}(\xi)(\cdot)$ has the property $(a)$. Moreover, we can define the multifunction $R_{1}: \operatorname{dom}(f) \cap \operatorname{clB}\left(x_{0}, \eta\right) \rightarrow 2^{L^{1}\left(\left[0, T_{0}\right], H\right)}$ as

$$
\begin{gathered}
R_{1}(\xi)=\left\{z \in S_{F\left(\cdot, x_{1}(\xi)(\cdot)\right.}^{1}:\left\|z(t)-r_{0}(\xi)(t)\right\| \leq k(t) \beta_{1}(t) \text { a.e. on }\left[0, T_{0}\right]\right\} \\
\text { for every } \xi \in \operatorname{dom}(f) \cap \operatorname{clB}\left(x_{0}, \eta\right),
\end{gathered}
$$

where

$$
\beta_{1}(t)=C \int_{0}^{t} \gamma(s) d s+T_{0}\left(\frac{\varepsilon}{2}+\frac{\varepsilon}{4}\right), \forall t \in\left[0, T_{0}\right]
$$

First, we observe that $R_{1}(\xi) \neq \emptyset, \forall \xi \in \operatorname{dom}(f) \cap c l B\left(x_{0}, \eta\right)$. In fact, using hypothesis 
$H(F)_{1}(j j)^{\prime}$ and the property $(i x)$ of Remark 1, we have

$$
d\left(r_{0}(\xi)(t), F\left(t, x_{1}(\xi)(t)\right)\right) \leq k(t) \beta_{1}(t), \text { a.e. on }\left[0, T_{0}\right] \text {. }
$$

Therefore, we can say that the multifunction $\Gamma_{1}(\xi):\left[0, T_{0}\right] \rightarrow 2^{H}$, defined by

$$
\Gamma_{1}(\xi)(t)=\left\{v \in F\left(t, x_{1}(\xi)(t)\right):\left\|v-r_{0}(\xi)(t)\right\| \leq k(t) \beta_{1}(t)\right\}, \forall t \in\left[0, T_{0}\right],
$$

has, for a.e. $t \in\left[0, T_{0}\right]$, nonempty values and thus, without any loss of generality, we can assume that $\Gamma_{1}$ has nonempty values. Moreover, $\Gamma_{1}(\xi)(\cdot)$ has measurable graph and so we are ready to apply theorem of [21] and we can deduce that there exists a measurable selection $z:\left[0, T_{0}\right] \rightarrow H$ of the multifunction $\Gamma_{1}(\xi)(\cdot)$. So, we conclude that $z \in R_{1}(\xi)$.

On the other hand, with analogous considerations made in order to obtain the function $r_{0}(\xi)(\cdot)$, we can get the function $r_{1}(\xi)(\cdot)$, selection of the multifunction

$$
t \rightarrow c l R_{1}(\xi)(t)=\left\{z \in S_{\left.F\left(\cdot, x_{1}(\xi)(\cdot)\right)^{:}\left\|z(t)-r_{0}(\xi)(t)\right\| \leq k(t) \beta_{1}(t)\right\}}\right.
$$

Easily, we can observe that $r_{1}(\xi)(\cdot)$ also satisfies the properties $(b),(c)$ and $(d)$.

Now, we suppose that we were able to produce $\left\{x_{k}(\xi)\right\}_{k=0}^{n}$ and $\left\{r_{k}(\xi)\right\}_{k=0}^{n}$ satisfying $(a) \rightarrow(d)$ above.

Let $x_{n+1}(\xi)(\cdot) \in C\left(\left[0, T_{0}\right], \Omega\right)$ be the unique solution of

$$
\left\{\begin{array}{c}
x^{\prime} \in-\partial^{-} f(x)+r_{n}(\xi), \text { a.e. }\left[0, T_{0}\right] \\
x(0)=\xi \in \operatorname{dom}(f) \cap \operatorname{clB}\left(x_{0}, \eta\right) .
\end{array}\right.
$$

Therefore, obviously, this function satisfies the condition $(a)$. As before, we get

$$
\begin{gathered}
\left\|x_{n+1}(\xi)(t)-x_{n}(\xi)(t)\right\| \\
\leq C \int_{0}^{t}\left\|r_{n}(\xi)(s)-r_{n-1}(\xi)(s)\right\| d s \leq C \int_{0}^{t} k(s) \beta_{n}(s) d s \\
=C \int_{0}^{t} k(s) \int_{0}^{s} \gamma(\tau) C^{n} \frac{(\theta(s)-\theta(\tau))^{n-1}}{(n-1) !} d \tau d s \\
+T_{0}\left(\sum_{k=0}^{n} \frac{\varepsilon}{\left.2^{k+1}\right) \int_{0}^{t} k(s) \frac{(\theta(s))^{n-1}}{(n-1) !} d s}\right. \\
=\int_{0}^{t} C^{n+1} k(s)\left(\int_{0}^{s} \gamma(\tau) \frac{(\theta(s)-\theta(\tau))^{n-1}}{(n-1) !} d \tau\right) d s \\
+T_{0}\left(\sum_{k=1}^{n} \frac{\varepsilon}{2^{k+1}}\right) \int_{0}^{t} k(s) \frac{(\theta(s))^{n}}{(n-1) !} d s \\
<\int_{0}^{t} C^{n+1} \gamma(\tau) \frac{(\theta(t)-\theta(\tau))^{n}}{n !} d \tau+T_{0}\left(\sum_{k=0}^{n+1} \frac{\varepsilon}{2^{k+1}}\right) \frac{(\theta(t))^{n}}{n !}=\beta_{n+1}(t)
\end{gathered}
$$




$$
\text { a.e. in }\left[0, T_{0}\right] \text {. }
$$

Therefore, using hypothesis $H(F)_{1}(j j)^{\prime}$, we have

$$
\begin{gathered}
d\left(r_{n}(\xi)(t), F\left(t, x_{n+1}(\xi)(t)\right) \leq k(t)\left\|x_{n}(\xi)(t)-x_{n+1}(\xi)(t)\right\|\right. \\
\leq k(t) \beta_{n+1}(t), \text { a.e. on }\left[0, T_{0}\right] .
\end{gathered}
$$

Now let $R_{n+1}$ : $\operatorname{dom}(f) \cap \operatorname{clB}\left(x_{0}, \eta\right) \rightarrow 2^{L^{1}\left(\left[0, T_{0}\right], H\right)}$ be the multifunction defined by

$$
\begin{aligned}
& R_{n+1}(\xi) \\
& \quad=\left\{z \in S_{F\left(\cdot, x_{n+1}(\xi)(\cdot)\right)}^{1}:\left\|z(t)-r_{n}(\xi)(t)\right\| \leq k(t) \beta_{n+1}(t) \text { a.e. in }\left[0, T_{0}\right]\right\} .
\end{aligned}
$$

From (3.23) above, we know that the multifunction $\Gamma_{n+1}(\xi):\left[0, T_{0}\right] \rightarrow 2^{H}$, defined by

$$
\Gamma_{n+1}(\xi)(t)=\left\{v \in F\left(t, x_{n+1}(\xi)(t)\right):\left\|v-r_{n}(\xi)(t)\right\|<k(t) \beta_{n+1}(t)\right\}
$$

is such that $\Gamma_{n+1}(\xi)(t) \neq \emptyset$ a.e. on $\left[0, T_{0}\right]$.

By modifying the above multifunction on a Lebesgue-null subset of $\left[0, T_{0}\right]$, we may assume without any loss of generality that $\Gamma_{n+1}(\xi)(t) \neq \emptyset$ for every $t \in\left[0, T_{0}\right]$. Also from Theorem 3.3 of [17], we know that $t \rightarrow F\left(t, x_{n+1}(\xi)(t)\right)$ is measurable (hence graph measurable), while $(t, v) \rightarrow\left\|v-r_{n}(\xi)(t)\right\|-k(t) \beta_{n+1}(t)=\gamma_{n+1}(\xi)(t, v)$ is clearly jointly measurable. So,

$$
G r \Gamma_{n+1}(\xi)=\left\{( v , t ) \in G r F ( \cdot , x _ { n + 1 } ( \xi ) ( \cdot ) : \gamma _ { n + 1 } ( \xi ) ( t , v ) < 0 \} \in \mathcal { L } \left(\left[0, T_{0}\right] \times B(H)\right.\right.
$$

with $\mathcal{L}\left(\left[0, T_{0}\right]\right)$ being the Lebesgue $\sigma$-field of $\left[0, T_{0}\right]$.

Applying Aumann's selection theorem, we get a measurable function $\tilde{z}:\left[0, T_{0}\right] \rightarrow H$ such that $\tilde{z}(t) \in \Gamma_{n+1}(\xi)(t), \quad \forall t \in\left[0, T_{0}\right], \quad$ so $\quad \tilde{z} \in R_{n+1}(\xi), \quad$ for every $\xi \in \operatorname{dom}(f) \cap \operatorname{cl} B\left(x_{0}, \eta\right)$. Moreover, $\xi \mapsto R_{n+1}(\xi)$ is (l.s.c. $)_{t}$ with decomposable values. Therefore, applying Theorem 3 of [4] to the multifunction $c l R_{n+1}(\cdot)$, we can get a continuous map $r_{n+1}: \operatorname{dom}(f) \cap \operatorname{clB}\left(x_{0}, \eta\right) \rightarrow L^{1}\left(\left[0, T_{0}\right], H\right)$ such that $r_{n+1}(\xi) \in \operatorname{cl} R_{n+1}(\xi)$, for every $\xi \in \operatorname{dom}(f) \cap \operatorname{clB}\left(x_{0}, \eta\right)$. Hence,e $r_{n+1}(\xi)(t) \in$ $F\left(t, x_{n+1}(\xi)(t)\right)$ a.e. on $\left[0, T_{0}\right]$ and $\left\|r_{n+1}(\xi)(t)-r_{n}(\xi)(t)\right\| \leq k(t) \beta_{n+1}(t)$ a.e. on $\left[0, T_{0}\right]$. Thus, by induction, we have produced the two sequences

$$
\left\{x_{n}(\xi)\right\}_{n \geq 0} \subseteq C\left(\left[0, T_{0}\right], \Omega\right) \text { and }\left\{r_{n}(\xi)\right\}_{n \geq 0} \subseteq L^{2}\left(\left[0, T_{0}\right], H\right)
$$

satisfying $(a) \rightarrow(d)$ above.

Then, using (3.22) we have

$$
\begin{gathered}
\int_{0}^{T_{0}}\left\|r_{n}(\xi)(t)-r_{n-1}(\xi)(t)\right\| d t \leq \int_{0}^{T_{0}} k(t) \beta_{n}(t) d t \\
\leq \int_{0}^{T_{0}} C^{n} \gamma(s) \frac{\left(\theta\left(T_{0}\right)\right)^{n}}{n !} d s+T_{0} \varepsilon \frac{\left(\theta\left(T_{0}\right)\right)^{n}}{n !} \\
\leq \frac{\left(\theta\left(T_{0}\right)\right)^{n}}{n !}\left[C^{n}\|\gamma\|_{1}+T_{0} \varepsilon\right] .
\end{gathered}
$$


So, from the above inequality, we deduce that $\left\{r_{n}(\xi)\right\}_{n}>0$ is a $L^{1}\left(\left[0, T_{0}\right], H\right)$ Cauchy sequence, uniformly in $\xi \in \operatorname{dom}(f) \cap \operatorname{cl} B\left(x_{0}, \eta\right)$. Also from $(a)$ we have

$$
\left\|x_{n+1}(\xi)-x_{n}(\xi)\right\|_{C\left(\left[0, T_{0}\right], \Omega\right)} \leq C\left\|r_{n+1}(\xi)-r_{n}(\xi)\right\|_{L^{1}\left(\left[0, T_{0}\right], H\right)}
$$

and, therefore, we can say that $\left\{x_{n}(\xi)\right\}_{n>0}$ is a Cauchy sequence in $C\left(\left[0, T_{0}\right], \Omega\right)$, uniformly in $\xi \in \operatorname{dom}(f) \cap \operatorname{cl} B\left(x_{0}, \eta\right)$.

Let $n \rightarrow \infty$ we have $x_{n+1}(\xi) \rightarrow x(\xi)$ in $C\left(\left[0, T_{0}\right], \Omega\right), r_{n}(\xi) \rightarrow r(\xi)$ in $L^{1}\left(\left[0, T_{0}\right], H\right)$ and both limits are continuous in $\operatorname{dom}(f) \cap \operatorname{clB}\left(x_{0}, \eta\right)$. Now let $y(\xi) \in C\left(\left[0, T_{0}\right], \Omega\right)$ be the unique solution of the problem

$$
\left\{\begin{array}{l}
z^{\prime} \in-\partial^{-} f(z)+r(\xi) \text { a.e. in }\left[0, T_{0}\right] \\
z(0)=\xi \in \operatorname{dom}(f) \cap \operatorname{clB}\left(x_{0}, \eta\right) .
\end{array}\right.
$$

By the property $(c)$, we have $r(\xi)(t) \in F(t, x(\xi)(t))$ a.e. As before, we have

$$
\left\|x_{n}(\xi)(t)-y(\xi)(t)\right\| \leq C \int_{0}^{t}\left\|r_{n-1}(\xi)(s)-r(\xi)(s)\right\| d s, \quad \forall t \in\left[0, T_{0}\right]
$$

by which we deduce that $x_{n}(\xi) \rightarrow y(\xi)$ in $C\left(\left[0, T_{0}\right], \Omega\right)$. Hence we have $x(\xi)=y(\xi)$, $\forall \xi \in \operatorname{dom}(f) \cap \operatorname{clB}\left(x_{0}, \eta\right)$.

Therefore, the function $u: \xi \mapsto x(\xi)$ is the desired selector of the multifunction $\xi \mapsto S(\xi)$.

\section{An Application: Existence of Periodic Solutions}

An immediate consequence of Theorem 5 is the following corollary:

Corollary 2: If hypotheses $H(f), H(F)_{1}$ hold, $\operatorname{dom}(f)$ is a closed subset of $\Omega$ and if there exists a compact and convex subset of $\operatorname{dom}(f) \cap \mathrm{clB}\left(x_{0}, \eta\right)$ such that $S(K)\left(T_{0}\right) \subseteq K$, where $\eta$ is fixed as in Theorem 5 , then there exists a solution $x(\cdot) \in$ $C\left(\left[0, T_{0}\right], \Omega\right)$ for the problem

$$
\left\{\begin{array}{c}
x^{\prime} \in-\partial^{-} f(x)+F(t, x) \text { a.e. on }\left[0, T_{0}\right] \\
x(0)=x\left(T_{0}\right)
\end{array}\right.
$$

Proof: Let $u: \operatorname{dom}(f) \cap \operatorname{cl} B\left(x_{0}, \eta\right) \rightarrow C\left(\left[0, T_{0}\right], \Omega\right)$ be the continuous selector of the multifunction $\xi \mapsto S(\xi)$ guaranteed by Theorem 5 . Let $e_{T_{0}}: C\left(\left[0, T_{0}\right], \Omega\right) \rightarrow H$ be the evaluation map, i.e. $e_{T_{0}}(x)=x\left(T_{0}\right)$.

Let $\widehat{u}=e_{T_{0}} \circ u: \operatorname{dom}(f) \cap \operatorname{cl} B\left(x_{0}, \eta\right) \rightarrow H$. we observe that the restriction of $\widehat{u}$ to the set $K$ assumes values in the set $K$, and moreover, $\widehat{u}: K \rightarrow K$ is a continuous and compact map. So Schauder's fixed point theorem gives us $\xi \in K$ such that $\xi=\widehat{u}(\xi)$.

Then $u(\xi)(\cdot) \in C\left(\left[0, T_{0}\right], \Omega\right)$ is the desired periodic trajectory. 


\section{References}

[1] Aubin, J.P. and Cellina, A., Differential Inclusions, Springer-Verlag, Berlin 1984.

[2] Aronszajn, N., Le correspondant topologique de l'unicité dans la théorie des équations differentielles, Ann. Math. 43 (1942), 730-738.

[3] Barbu, V., Nonlinear Semigroups and Differential Equations in Banach Space, Noordhoff International Publ, Ledyen, The Netherlands 1976.

[4] Bressan, A. and Colombo, G., Extension and selections of maps with decomposable values, Studia Math. 90 (1988), 69-86.

[5] Brezis, H., Operateurs Maximaux Monotones et semi-groupes de contractions dans les espace de Hilbert, North-Holland, Amsterdam 1973.

[6] Cardinali, T., Colombo, F. Papalini, F. and Tosques, M., On a class of evolution equations without convexity, Nonlinear Analysis 28 (1997), 217-234.

[7] Cardinali, T. and Papalini, F., Convergence results for nonlinear evolution inclusions, Disc. Math.-Diff. Incl. 15 (1995), 43-60.

[8] Colombo, G. and Tosques, M., Multivalued perturbations for a class of nonlinear evolution equations, Ann. Mat. Pura e Appl. 4 (1991), 147-162.

[9] DeBlasi, F., Characterizations of certain classes of semicontinuous multifunctions by continuous approximations, J. Math. Anal. Appl. 106 (1985), 1-18.

[10] De Blasi, F. and Myjak, J., On the solution sets for differential inclusions, Bull. Pol. Acad. Sci. 33 (1985), 17-23.

[11] Fan, K., Fixed point and minimax theorems in locally convex topological linear spaces, Proc. Nat. Acad. Sci. Usa 38 (1952), 121-126.

[12] Fryszkowski, A., Continuous selections for a class of nonconvex multivalued maps, Studia Math. 78 (1983), 163-174.

[13] Himmelberg, C.J. and Van Vleck, F.S., A note on solution sets of differential inclusions, Rocky Mountain J. Math. 12 (1982), 621-625.

[14] $\mathrm{Hu}, \mathrm{S}$. and Papageorgiou, N.S., Time-dependent subdifferential evolution inclusions and optimal control, to appear.

[15] Hyman, D., On decreasing sequences of compact absolute retracts, Fundamenta Math. 64 (1969), 91-97.

[16] Papageorgiou, N.S., Convergence theorems for Banach space valued integrable multifunctions, Inter. J. Math. and Math. Sci. 10 (1987), 433-442.

[17] Papageorgiou, N.S., On measurable multifunctions with applications to random multivalued equations, Math. Japonica 32 (1987), 437-464.

[18] Papageorgiou, N.S. and Papalini, F., On the structure of the solution set of evolution inclusions with time-dependent subdifferentials, to appear.

[19] Papalini, F., Properties of the solution set of evolution inclusions, Nonlinear Anal. 26 (1996), 1279-1292.

[20] Tosques, M., Quasi-autonomous parabolic evolution equations associated with a class of nonlinear operators, Ricerche di Mat. 38 (1989), 63-92.

[21] Wagner, D., Survey of measurable selection theorems, SIAM J. Control Optim. 15 (1977), 859-903. 


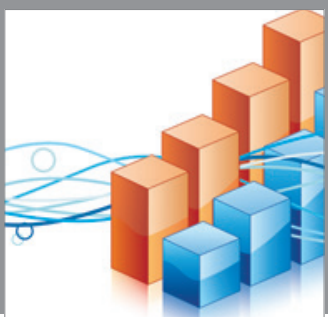

Advances in

Operations Research

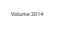

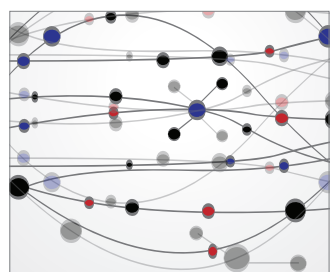

\section{The Scientific} World Journal
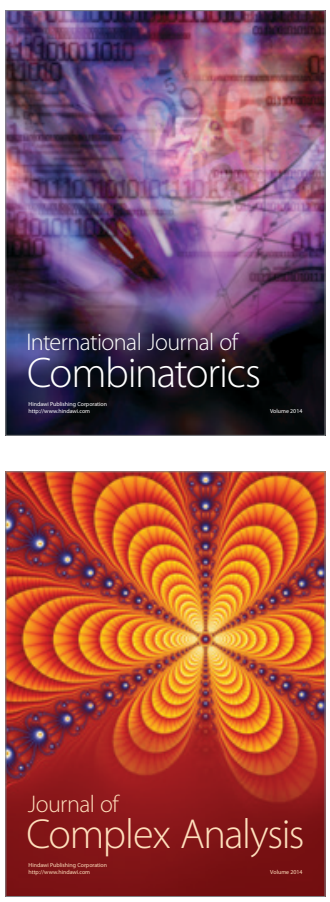

International Journal of

Mathematics and

Mathematical

Sciences
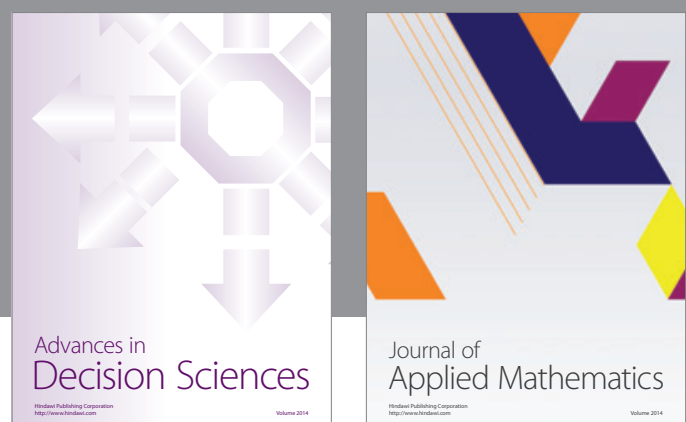

Journal of

Applied Mathematics
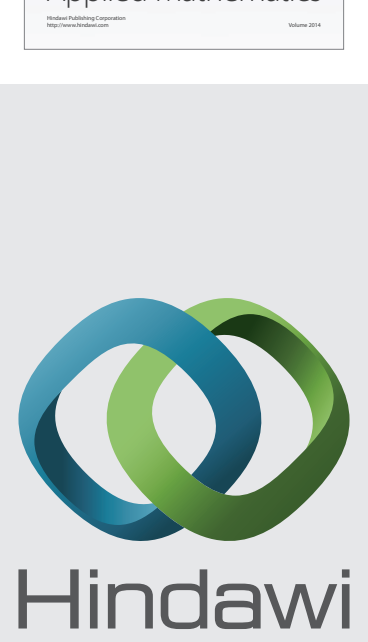

Submit your manuscripts at http://www.hindawi.com
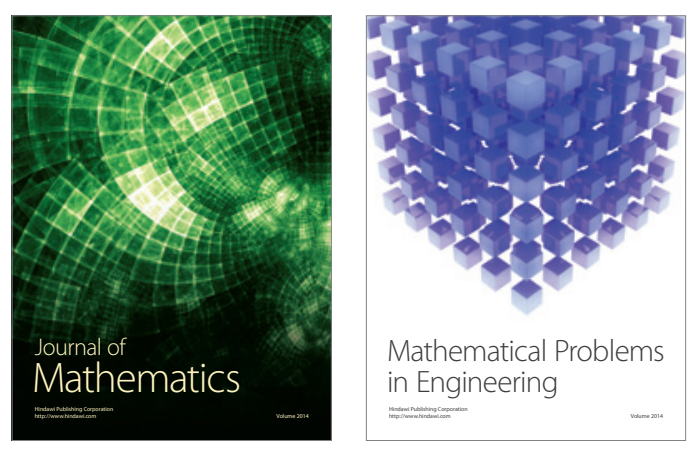

Mathematical Problems in Engineering
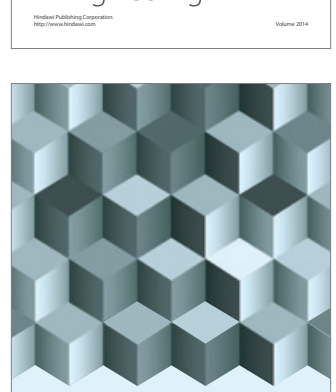

Journal of

Function Spaces
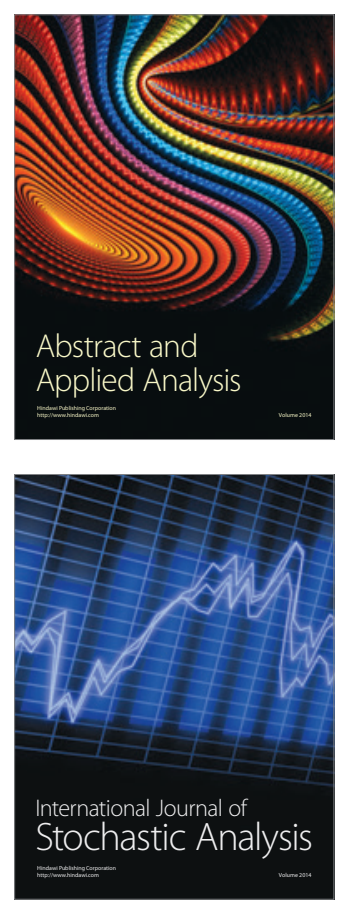

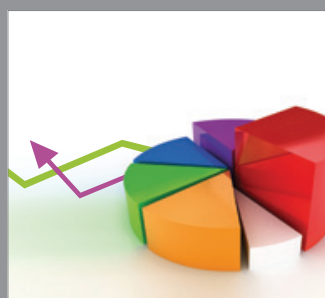

ournal of

Probability and Statistics

Promensencen
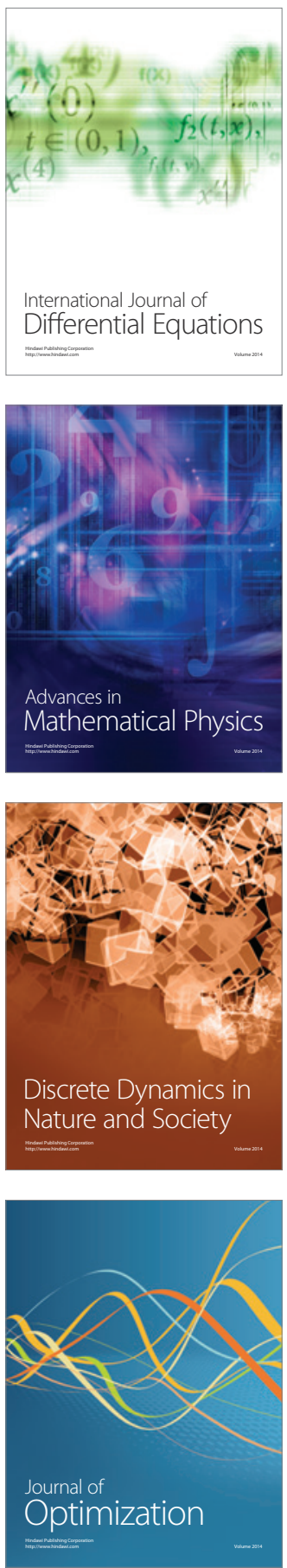